\title{
Fractional diffusion limit for collisional kinetic equations
}

\author{
Antoine Mellet ${ }^{1}$, Stéphane Mischler ${ }^{2}$ and Clément Mouhot ${ }^{2}$
}

\begin{abstract}
This paper is devoted to diffusion limits of linear Boltzmann equations. When the equilibrium distribution function is a Maxwellian distribution, it is well known that for an appropriate time scale, the small mean free path limit gives rise to a diffusion equation. In this paper, we consider situations in which the equilibrium distribution function is a heavy-tailed distribution with infinite variance. We then show that for an appropriate time scale, the small mean free path limit gives rise to a fractional diffusion equation.
\end{abstract}

Mathematics Subject Classification (2000): 76P05 Rarefied gas flows, Boltzmann equation [See also 82B40, 82C40, 82D05], 26A33 Fractional derivatives and integrals.

Keywords: fractional diffusion, fractional heat equation, anomalous heat transport, linear Boltzmann equation, relaxation equation, linear BGK equation, diffusion limit, anomalous diffusion limit, anomalous diffusive time scale, mathematical derivation.

Acknowledgments: Antoine Mellet gratefully thanks the CEREMADE at the Université Paris Dauphine, where most of this research was performed, for its hospitality. Antoine Mellet was also partially supported by NSERC Grant 341253-07.

\section{Contents}

\section{Introduction}

\footnotetext{
${ }^{1}$ Email: mellet@math.umd.edu

Department of Mathematics, University of Maryland, College Park, MD 20742 USA

${ }^{2}$ Emails: mischler@ceremade.dauphine.fr, mouhot@ceremade.dauphine.fr

CEREMADE, Université Paris Dauphine, Place du Maréchal de Lattre de Tassigny, 75775

Paris cedex 16, FRANCE
} 
2 Proof of Theorem 1.1 (simplest scenario) 8

3 Diffusion limit results for general collision operator 12

4 Proof of Theorem $3.2 \quad 19$

4.1 A priori estimates . . . . . . . . . . . . . 19

4.2 Another formulation of the rescaled equation . . . . . . . 22

4.3 Passing to the limit in $a^{\varepsilon} \ldots \ldots \ldots \ldots$. . . . . . . . 24

4.4 The remaining term . . . . . . . . . . . . . . 28

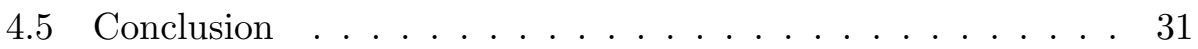

5 Proof of Theorem 3.4

6 Appendix $\quad 36$

\section{Introduction}

It is well known that under appropriate scaling, the asymptotic analysis of collisional kinetic equations can lead to diffusion-type equations. This scaling corresponds to a long time scale and a small mean-free path. More precisely, the starting point is the following collisional kinetic equation:

$$
\begin{array}{ll}
\partial_{t} f+v \cdot \nabla_{x} f=L(f) & \text { in }(0, \infty) \times \mathbb{R}^{N} \times \mathbb{R}^{N}, \\
f(0, .)=f_{0} & \text { in } \mathbb{R}^{N} \times \mathbb{R}^{N},
\end{array}
$$

which models the evolution of a particle distribution function, $f(t, x, v) \geq 0$ depending on the time $t>0$, the position $x \in \mathbb{R}^{N}$ and a variable $v \in$ $\mathbb{R}^{N}$. This variable $v$ usually represents the velocity of the particles, or some other internal degree of freedom of the particles, such as a wave vector. For simplicity, we take $v \in \mathbb{R}^{N}$, though other spaces could be considered (torus for the wave vector in semiconductor or bounded set for relativistic particles).

We then introduce the macroscopic variables

$$
x^{\prime}=\varepsilon x \quad t^{\prime}=\theta(\varepsilon) t
$$

and the rescaled distribution function

$$
f^{\varepsilon}\left(t^{\prime}, x^{\prime}, v\right)=f(t, x, v)
$$


where $\varepsilon$ is a small parameter. The function $f^{\varepsilon}$ is now solution of (we have skipped the primes)

$$
\theta(\varepsilon) \partial_{t} f^{\varepsilon}+\varepsilon v \cdot \nabla_{x} f^{\varepsilon}=L\left(f^{\varepsilon}\right) .
$$

The object of this paper is to investigate the behavior, as $\varepsilon$ goes to zero, of the solutions of (3). It will naturally strongly depend on the properties of the collision operator $L$.

Throughout this paper, we will assume that $L$ is a linear Boltzmann operator (sometimes also called "scattering" operator), describing the interactions of the particles with the surrounding medium, of the form:

$$
L(f)=\int_{\mathbb{R}^{N}}\left[\sigma\left(v, v^{\prime}\right) f\left(v^{\prime}\right)-\sigma\left(v^{\prime}, v\right) f(v)\right] d v^{\prime}
$$

with a non-negative collision kernel $\sigma=\sigma\left(v, v^{\prime}\right) \geq 0$. The operator $L$ is conservative, i.e. it preserves the total mass of the distribution. Under classical assumptions on the collision kernel $\sigma$, there exists a unique positive normalized equilibrium function $F$ :

$$
F=F(v)>0 \text { a.e. on } \mathbb{R}^{N}, \quad \int_{\mathbb{R}^{N}} F(v) d v=1 \text { and } L(F)=0 .
$$

In the sequel, we always assume that $F$ exists and is an even function of $v$.

The derivation of diffusion-type equations from kinetic equations such as (3) was first investigated by E. Wigner [20, A. Bensoussan, J.L. Lions and G. Papanicolaou in [2] and E.W. Larsen and J.B. Keller [14]. In [7], P. Degond, T. Goudon and F. Poupaud consider very general collision operators of the form (4). When $F$ decreases "quickly enough" for large values of $|v|$ (and under a few additional assumptions on $\sigma$ and $F$ ), they prove in particular that for $\theta(\varepsilon)=\varepsilon^{2}, f^{\varepsilon}(t, x, v)$ converges, when $\varepsilon$ goes to zero, to a function of the form $\rho(t, x) F(v)$ where the density $\rho(t, x)$ solves a diffusion equation

$$
\partial_{t} \rho-\nabla_{x}\left(D \nabla_{x} \rho\right)=0,
$$

with diffusion matrix $D$ given by the following formula

$$
D=\int_{\mathbb{R}^{N}}(v \otimes \chi) d v \quad \text { with } \quad L(\chi)=-v F .
$$

In order to introduce our problem in simple terms, we now consider, in this introduction and in the next section, that $L$ has the following very simple form:

$$
L(f)=\rho F-f, \quad \rho=\langle f\rangle:=\int_{\mathbb{R}^{N}} f(v) d v
$$


which corresponds to the choice of a collision kernel $\sigma\left(v, v^{\prime}\right)=F(v)$. This baby model is usually called "linear relaxation" or sometimes "linear BGK" collision operator. We shall come back to more general (and realistic) collision kernel $\sigma\left(v, v^{\prime}\right)$ in Section 3. Under (7), it is readily seen that we can take $\chi=v F$ in the definition of the diffusion matrix $D$ and thus:

$$
D=\int_{\mathbb{R}^{N}}(v \otimes v) F(v) d v .
$$

In particular, in order for $D$ to be finite, we need the second moment of $F$ to be finite:

$$
\int_{\mathbb{R}^{N}}|v|^{2} F(v) d v<\infty
$$

This is the case, for example, when the equilibrium $F$ is given by the socalled "Maxwellian" distribution $F(v)=C \exp \left(-\frac{v^{2}}{2}\right)$. The main result of this paper can then be summarized as follows: If $F$ is such that (8) does not hold, then, under appropriate time scale, the limit $\varepsilon \rightarrow 0$ in (3) leads to a fractional diffusion equation instead of (5).

More precisely, we will assume that $F$ is a heavy-tailed distribution functions, that is typically (we will consider slightly more general $F$ later on):

$$
F(v) \sim \frac{\kappa_{0}}{|v|^{N+\alpha}} \quad \text { as } \quad|v| \rightarrow \infty
$$

with $\kappa_{0}>0$ and $\alpha>0$ (this last condition guarantees that $F$ is integrable).

Heavy-tailed distribution functions arise in many contexts. For instance, most astrophysical plasmas are observed to have velocity distribution functions exhibiting power law tails (see Summers and Thorne [18] or Mendis and Rosenberg [16]). Dissipative collision mechanisms in granular gases can also produce power law tails: see for instance [11] for the so-called "inelastic Maxwell model" introduced in [4]. One can also refers to the more general review paper [19]. We also mention that a recent work [5] has shown that even elastic collision mechanisms can produce power law tail behaviors in the case of mixture of gases with Maxwellian collision kernel. Power law tails are also common in economy where they are referred to as Pareto distributions (as well as in statistics and probability more generally). Here are some samples of (very different) mathematical works using statistical physics models to account for these power laws in economy: Newman [17, Duering-Toscani [10] and Wright [21]. Let us just mention to the reader that, in the case of economy, the interpretation of these power law distributions as equilibria of statistical physics models (which is the core of the 
field now known as "econophysics") is controversial since it does not take into account any individual rationality.

It is thus the goal of this paper to investigate what happens to diffusion limits when the velocity repartition is no longer described by a Gaussian function. More precisely we shall be particularly interested in those heavytailed distributions whose variance is infinite, since in this case we shall show that the diffusion limit yields equations of the form (11), and our underlining motivation was indeed to provide a microscopic derivation for these fractional diffusion equations, which is lacking at now.

As a striking link between these goal and motivation, let us mention that a famous case of such distribution with infinite variance are the stable (or Lévy) distributions. And the latter plays an important role in probability theory since it can be interpreted as the law of a "Lévy walk" whose law evolution is governed by a fractional diffusion equation. To say it differently and in a more analytical way, these stable distributions are the fundamental solutions of the fractional diffusion equation. They thus play the role played by the gaussian distribution in the case of the heat equation.

When (9) holds with $\alpha>2$ then (8) is still satisfied, and the analysis leading to (5) can be performed. We are thus interested in values of $\alpha$ less than 2. More precisely, when $\alpha \in(0,2)$, we will prove that the appropriate diffusion scaling is given by

$$
\theta(\varepsilon):=\varepsilon^{\alpha},
$$

and that the solution $f^{\varepsilon}$ of (3) then converges to $\rho(t, x) F(v)$ with $\rho$ solution of the following fractional diffusion equation

$$
\begin{array}{ll}
\partial_{t} \rho+\kappa\left(-\Delta_{x}\right)^{\alpha / 2} \rho=0 & \text { in }(0, \infty) \times \mathbb{R}^{N}, \\
\rho(0, .)=\rho_{0} & \text { in } \mathbb{R}^{N},
\end{array}
$$

with

$$
\kappa=\int_{\mathbb{R}^{N}} \frac{w_{1}^{2}}{1+w_{1}^{2}} \frac{\kappa_{0}}{|w|^{N+\alpha}} d w
$$

We recall that the operator $\left(-\Delta_{x}\right)^{\alpha / 2}$ denotes the fractional operator, defined for instance by the Fourier formula

$$
\left(-\Delta_{x}\right)^{\alpha / 2} \rho:=\mathcal{F}^{-1}\left(|k|^{\alpha} \mathcal{F}(\rho)(k)\right),
$$

where $\mathcal{F}$ stands for the Fourier transform in the space variable. This operator has a lot of nice properties, similar to that of the usual Laplace operator, most notably it retains some ellipticity (in the sense that its $L^{2}$ Dirichlet 
form controls some fractional Sobolev norm $H^{\alpha / 2}$ ). The fundamental difference is the fact that this operator is nonlocal for any $0<\alpha<2$.

Before we state our first result, let us introduce some functional spaces definitions. We denote by $L^{p}, p \in[1,+\infty]$ the usual Lebesgue space on $\mathbb{R}^{N}$ or $\mathbb{R}^{N} \times \mathbb{R}^{N}$ (this will always be clear from the context), with the flat measure. When $\Theta>0$ is a given positive locally locally integrable weight function, we write $L^{p}(\Theta)$ for the Lebesgue space with measure $\Theta d x$, that is the Banach space defined by the norm

$$
\|f\|_{L^{p}(\Theta)}:=\left(\int_{\mathbb{R}^{N}}|f|^{p} \Theta\right)^{1 / p} .
$$

We shall sometimes use subscript letters in order to recall and emphasize which variable we are considering for the Lebesgue space.

Our first result could thus read as follows:

Theorem 1.1. Assume that $L$ is given by (7) and that $F$ satisfies (9) with $\alpha \in(0,2)$. Assume furthermore that $f_{0} \in L^{2}\left(F^{-1}\right)$ and let $f^{\varepsilon}$ be the solution of (3) with $\theta(\varepsilon)=\varepsilon^{\alpha}$ and initial condition $f_{0}$.

Then, when $\varepsilon$ goes to zero, $f^{\varepsilon}$ converges in $L^{\infty}\left(0, T ; L^{2}\left(\mathbb{R}^{N} \times \mathbb{R}^{N}\right)\right)$-weak to $\rho F$ with $\rho=\rho(t, x)$ the unique solution to the fractional diffusion equation (11), (12), (13).

Note that under (9), $\alpha$ can be characterized as follows:

$$
\alpha=\sup \left\{a \geq 0 ; \int_{\mathbb{R}^{N}}|v|^{a} F(v) d v<\infty\right\} .
$$

This characterization will be more obvious in Section 3 when we add to $F$ a slowly varying function. In particular, we will see that the fact that the moment of order $\alpha$ is finite or not does not affect the asymptotic equation.

It is also worth pointing out that when $L$ is given by (7), fractional diffusion is only observed when the moment of order 2 is unbounded, i.e. when the energy associated to the equilibrium distribution function $F$ is infinite. We will see, however, in Theorem 3.2 that fractional diffusion may arise even when the energy of $F$ (and higher order moments) is finite, provided the collision frequency

$$
\nu(v)=\int_{\mathbb{R}^{N}} \sigma\left(v^{\prime}, v\right) d v^{\prime}
$$

in (44) is degenerate for high velocities: $\nu(v) \sim|v|^{\beta}$ for large $v$ with $\beta<0$ small enough (for instance with $\alpha>2$ and $\beta<2-\alpha<0$ ). 
Conversely, even when the energy of the equilibrium if infinite, it is possible in some cases to recover a classical diffusion limit. This is the case for instance when $\alpha \in(1,2)$ and $2-\alpha<\beta<1$, or $\alpha \in(0,1)$ and $1<\beta<2-\alpha$ (see Theorem [3.6). These ranges of the parameters $\beta$ are quite surprising as well as the fact that $\gamma(\alpha, \beta)$ (the order of the limit fractional diffusion operator) is decreasing (to 1 ) as a function of $\beta$ when $\alpha \in(0,1)$ and increasing (to 1 ) as a function of $\beta$ when $\alpha \in(1,2)$, whereas one would expect at first guess from physics that the stronger $\beta$, the stronger the diffusion at the limit as it enhances collisions for high velocities. This calls for a satisfying physical interpretation.

Theorem 1.1 states that in the long time limit, the particles evolve according to an anomalous diffusion process (one calls a diffusion process anomalous if the mean square displacement grows like a nonlinear function of the time variable in the long time limit). Anomalous diffusion limits for kinetic models are well known in the case of a gas confined between two plates, when the distance between the plates goes to 0 (see [6], [12], [8], [9]). In that case, the limiting equation is still a standard diffusion equation, but the time scale is anomalous $\left(\theta(\varepsilon) \sim \varepsilon^{2} \ln \left(\varepsilon^{-1}\right)\right)$. The particles travelling in directions nearly parallel to the plates are responsible for the anomalous scaling. We will see that a similar behavior arises here when $\alpha=2$ in (9) (i.e. when the second moment is unbounded, but all moments of smaller orders are bounded), see Theorem 3.6.

A fractional diffusion equation has been obtained as a diffusive limit from a linear phonon-Boltzmann equation simultaneously and independently by M. Jara, T. Komorowski and S. Olla in [13], by a different probability approach. Let us also mention a work in progress [15] by B. Texier and the first author, where a derivation of fractional diffusion equations from kinetic models is obtained in the framework of a gas confined between two plates with singular equilibrium distribution functions, by analytic means.

Our approach relies on the use of Laplace-Fourier transforms and a careful computation of the asymptotic behavior of the symbol of the differential operator. This approach is quite simple and allows for an explicit computation of the coefficients of the asymptotic equation under optimal assumptions. Preliminary computations seem to indicate that it can generalize to equations involving an external force field, but it seems harder to generalize to a non-linear collision kernel (Pauli's statistic for instance). A more "nonlinear" approach, as was the moments method in the classical diffusion case, would be welcomed.

Let us now briefly outline the contents of the paper: The proof of The- 
orem 1.1. which corresponds to the simplest case, is presented in the next section. In Section 3, we state our main result (Theorem 3.2) which generalizes Theorem 1.1 to a large class of linear collision operator $L$ of the form (4). We also address the critical case $(\alpha=2)$ (Theorem 3.4) and the classical diffusion case in Theorem 3.6. Theorem 3.2 (as well as Theorem 3.6) are then proven in Section 4 while Section 5 is devoted to the proof of Theorem 3.4.

\section{Proof of Theorem 1.1 (simplest scenario)}

The goal of the section is to present the main ideas of the proof, which are in fact quite simple. We thus make all sort of simplifying assumptions in order to focus on the important aspect of the proofs. We recall that $L$ is given by (7), and we assume, instead of (9), that the equilibrium function $F$ satisfies:

$$
F(v) \leq \kappa_{0}|v|^{-N-\alpha} \text { for all } v \in \mathbb{R}^{N}, \quad F(v)=\kappa_{0}|v|^{-N-\alpha} \quad \text { if } \quad|v| \geq 1,
$$

with $\kappa_{0}>0$.

The proof is divided in 4 steps. Note that the proof of the main result in Section 4 will follow exactly the same steps.

Step 1: A priori estimates for $f^{\varepsilon}$.

The solution $f^{\varepsilon}$ of (3) (with $\theta(\varepsilon)=\varepsilon^{\alpha}$ ) satisfies

$$
\begin{aligned}
\varepsilon^{\alpha} \frac{d}{d t} \int_{\mathbb{R}^{2 N}} \frac{\left(f^{\varepsilon}\right)^{2}}{2} F^{-1} d v d x & =\int_{\mathbb{R}^{2 N}} L\left(f^{\varepsilon}\right) f^{\varepsilon} F^{-1} d v d x \\
& =\int_{\mathbb{R}^{2 N}}\left[\left(\rho^{\varepsilon}\right)^{2} F-\left(f^{\varepsilon}\right)^{2} F^{-1}\right] d v d x \\
& =-\int_{\mathbb{R}^{2 N}}\left[f^{\varepsilon}-\rho^{\varepsilon} F\right]^{2} F^{-1} d v d x,
\end{aligned}
$$

from which we deduce the two estimates

$$
\sup _{t \geq 0} \int_{\mathbb{R}^{2 N}} \frac{\left(f^{\varepsilon}(t, .)\right)^{2}}{F} d v d x \leq \int_{\mathbb{R}^{2 N}} \frac{f_{0}^{2}}{F} d v d x=\left\|f_{0}\right\|_{L^{2}\left(F^{-1}\right)},
$$

and

$$
\int_{0}^{\infty} \int_{\mathbb{R}^{2 N}}\left[f^{\varepsilon}-\rho^{\varepsilon} F\right]^{2} F^{-1} d v d x d t \leq \frac{\varepsilon^{\alpha}}{2}\left\|f_{0}\right\|_{L^{2}\left(F^{-1}\right)} .
$$

Cauchy-Schwarz inequality also gives:

$$
\rho^{\varepsilon}(t, x)=\int_{\mathbb{R}^{N}} \frac{f^{\varepsilon}}{F^{1 / 2}} F^{1 / 2} d v \leq\left(\int_{\mathbb{R}^{N}} \frac{\left(f^{\varepsilon}\right)^{2}}{F} d v\right)^{1 / 2},
$$


so that $\rho^{\varepsilon}(t, x)$, as well as $L\left(f^{\varepsilon}\right)$, are well defined a.e., and

$$
\sup _{t \geq 0} \int_{\mathbb{R}^{N}} \rho^{\varepsilon}(t, .)^{2} d x \leq\left\|f_{0}\right\|_{L^{2}\left(F^{-1}\right)} .
$$

\section{Step 2: Another formulation of the rescaled equation.}

We denote by $\widehat{f^{\varepsilon}}$ the Laplace-Fourier transform of $f^{\varepsilon}$ with respect to $t$ and $x$, defined by

$$
\widehat{f^{\varepsilon}}(p, k, v)=\int_{\mathbb{R}^{N}} \int_{0}^{\infty} e^{-p t} e^{-i k \cdot x} f^{\varepsilon}(t, x, v) d t d x, \quad p>0, k \in \mathbb{R}^{N} .
$$

The function $\widehat{f^{\varepsilon}}$ then satisfies

$$
\left.\varepsilon^{\alpha} p \widehat{f^{\varepsilon}}-\varepsilon^{\alpha} \widehat{f_{0}}+\varepsilon i v \cdot k \widehat{f \varepsilon}=\widehat{\left\langle f^{\varepsilon}\right.}\right\rangle F-\widehat{f^{\varepsilon}},
$$

where $\widehat{f_{0}}$ denotes the Fourier transform of the initial datum $f_{0}$. We can rewrite this equality as

$$
\widehat{f \varepsilon}=\frac{F}{1+\varepsilon^{\alpha} p+\varepsilon i v \cdot k} \widehat{\rho^{\varepsilon}}+\frac{\varepsilon^{\alpha} \widehat{f_{0}}}{1+\varepsilon^{\alpha} p+\varepsilon i v \cdot k},
$$

with $\widehat{\rho^{\varepsilon}}(p, k)=\left\langle\widehat{f^{\varepsilon}}\right\rangle(p, k)$ the Laplace-Fourier transform of $\rho^{\varepsilon}$, and integrating this equality with respect to $v$, we obtain:

$$
\widehat{\rho^{\varepsilon}}=\left(\int_{\mathbb{R}^{N}} \frac{F(v)}{1+\varepsilon^{\alpha} p+\varepsilon i v \cdot k} d v\right) \widehat{\rho^{\varepsilon}}+\left(\int_{\mathbb{R}^{N}} \frac{\varepsilon^{\alpha} \widehat{f}_{0}}{1+\varepsilon^{\alpha} p+\varepsilon i v \cdot k} d v\right) .
$$

The normalization condition for $F$ now yields:

$$
\int_{\mathbb{R}^{N}} \frac{\widehat{f_{0}}}{1+\varepsilon^{\alpha} p+\varepsilon i v \cdot k} d v+a^{\varepsilon} \widehat{\rho^{\varepsilon}}=0,
$$

with

$$
a^{\varepsilon}(p, k):=\frac{1}{\varepsilon^{\alpha}} \int_{\mathbb{R}^{N}}\left(\frac{1}{1+\varepsilon^{\alpha} p+\varepsilon i v \cdot k}-1\right) F(v) d v .
$$

Let us prove that that the first term converges to $\widehat{\rho_{0}}$ when $\varepsilon$ goes to zero. The assumption $f_{0} \in L^{2}\left(F^{-1}\right)$ implies in particular that $f_{0} \in L_{x}^{2}\left(L_{v}^{1}\right)$. Hence its the Fourier transform $\widehat{f}_{0}$ also belongs to $L_{k}^{2}\left(L_{v}^{1}\right)$ by Parseval equality, which means that $\widehat{f}_{0}$ is integrable in $v$ for almost all $k$. This allows to apply the Lebesgue dominated convergence theorem, which yields, for almost every $k$,

$$
\int_{\mathbb{R}^{N}} \frac{\widehat{f_{0}}}{1+\varepsilon^{\alpha} p+\varepsilon i v \cdot k} d v \longrightarrow \int_{\mathbb{R}^{N}} \widehat{f}_{0} d v=\widehat{\rho}_{0} .
$$


So we are left with the task of studying the limit, as $\varepsilon$ goes to zero, of the coefficient $a^{\varepsilon}$.

Step 3: The cornerstone argument of the proof: where fractional diffusion symbol appears.

A simple computation leads to

$$
\begin{aligned}
a^{\varepsilon}(p, k)= & -\frac{1}{\varepsilon^{\alpha}} \int_{\mathbb{R}^{N}} \frac{\varepsilon^{\alpha} p+\varepsilon i v \cdot k}{1+\varepsilon^{\alpha} p+\varepsilon i v \cdot k} F(v) d v \\
= & -p \int_{\mathbb{R}^{N}} \frac{1+\varepsilon^{\alpha} p}{\left(1+\varepsilon^{\alpha} p\right)^{2}+\varepsilon^{2}(v \cdot k)^{2}} F(v) d v \\
& -\frac{1}{\varepsilon^{\alpha}} \int_{\mathbb{R}^{N}} \frac{(\varepsilon v \cdot k)^{2}}{\left(1+\varepsilon^{\alpha} p\right)^{2}+\varepsilon^{2}(v \cdot k)^{2}} F(v) d v
\end{aligned}
$$

(the term involving $\varepsilon i v \cdot k$ on the numerator vanishes thanks to the symmetry of $F$ ). The first term in the right hand side is bounded by $|p|$ (uniformly in $\varepsilon)$ and the dominated convergence theorem readily implies that it converges to $-p \int_{\mathbb{R}^{N}} F(v) d v=-p$ as $\varepsilon$ goes to zero. So it only remains to study

$$
d^{\varepsilon}(p, k):=\int_{\mathbb{R}^{N}} \frac{\varepsilon^{2-\alpha}(v \cdot k)^{2}}{\left(1+\varepsilon^{\alpha} p\right)^{2}+\varepsilon^{2}(v \cdot k)^{2}} F(v) d v .
$$

If the second moment of $F$ is bounded, then it is readily seen that with $\alpha=2$, we have

$$
d^{\varepsilon}(p, k) \longrightarrow \int_{\mathbb{R}^{N}}(v \cdot k)^{2} F(v) d v=\kappa|k|^{2}, \quad \kappa \in(0, \infty),
$$

and the limit $\varepsilon \rightarrow 0$ in (10) leads to the diffusion equation (5).

With (15) and $\alpha \in(0,2)$, the second moment of $F$ is unbounded. We then claim that for any $p \geq 0, k \in \mathbb{R}^{N}$, we have:

$$
\left|d^{\varepsilon}(p, k)\right| \leq \kappa|k|^{\alpha} \quad \text { and } \quad d^{\varepsilon}(p, k) \underset{\varepsilon \rightarrow 0}{\longrightarrow} \kappa|k|^{\alpha},
$$

with $\kappa \in(0, \infty)$ given by (13).

As a matter of fact, the first inequality in (20) follows from (15):

$$
0 \leq d^{\varepsilon}(p, k) \leq \int_{\mathbb{R}^{N}} \frac{\varepsilon^{2-\alpha}(v \cdot k)^{2}}{1+\varepsilon^{2}(v \cdot k)^{2}} \frac{\kappa_{0}}{|v|^{N+\alpha}} d v=\kappa|k|^{\alpha},
$$

where the last equality is obtained by making the change of variables $w:=$ $\varepsilon|k| v$. And in order to get the convergence of $d^{\varepsilon}$, we simply write

$$
d^{\varepsilon}(p, k)=d_{1}^{\varepsilon}(p, k)+d_{2}^{\varepsilon}(p, k)
$$


with

$$
\begin{aligned}
d_{1}^{\varepsilon}(p, k) & =\int_{|v| \leq 1} \frac{\varepsilon^{2-\alpha}(v \cdot k)^{2}}{\left(1+\varepsilon^{\alpha} p\right)^{2}+\varepsilon^{2}(v \cdot k)^{2}} F(v) d v \\
& \leq \varepsilon^{2-\alpha} \int_{|v| \leq 1}(v \cdot k)^{2} F(v) d v \\
& \leq \varepsilon^{2-\alpha}|k|^{2} \int_{|v| \leq 1} F(v) d v \rightarrow 0
\end{aligned}
$$

and

$$
\begin{aligned}
d_{2}^{\varepsilon}(p, k) & =\int_{|v| \geq 1} \frac{\varepsilon^{2-\alpha}(v \cdot k)^{2}}{\left(1+\varepsilon^{\alpha} p\right)^{2}+\varepsilon^{2}(v \cdot k)^{2}} \frac{\kappa_{0}}{|v|^{N+\alpha}} d v \\
& =|k|^{\alpha} \int_{|w| \geq \varepsilon|k|} \frac{w_{1}^{2}}{\left(1+\varepsilon^{\alpha} p\right)^{2}+w_{1}^{2}} \frac{\kappa_{0}}{|w|^{N+\alpha}} d w \rightarrow \kappa|k|^{\alpha},
\end{aligned}
$$

where we make again the change of variables $w:=\varepsilon|k| v$ and use the dominated convergence theorem. We have thus shown:

Proposition 2.1. If $\alpha \in(0,2)$, then

$$
a^{\varepsilon}(p, k) \longrightarrow-p-\kappa|k|^{\alpha} \quad \text { as } \varepsilon \rightarrow 0
$$

with $\kappa \in(0, \infty)$ given by (13). Furthermore, $a^{\varepsilon}(p, k)$ satisfies

$$
\left|a^{\varepsilon}(p, k)\right| \leq|p|+\kappa|k|^{\alpha} .
$$

\section{Step 4: Conclusion.}

From the bound (18) and up to extraction of a subsequence, we know that there exists $\eta \in L^{\infty}\left(0, \infty ; L^{2}\left(\mathbb{R}^{N}\right)\right)$ such that $\rho^{\varepsilon} \rightarrow \eta$ weakly in $L^{\infty}(0, \infty$; $\left.L^{2}\left(\mathbb{R}^{N}\right)\right)$. On the one hand, from (18) again, we have $\widehat{\rho^{\varepsilon}}$ bounded in $L^{\infty}\left(a, \infty ; L^{2}\left(\mathbb{R}^{N}\right)\right)$ for any $a>0$. On the other hand, for any $\varphi=\varphi(t) \in$ $\mathcal{D}(0, \infty)$ its Laplace transform $\mathcal{L} \varphi$ belongs to $L^{1}(0, \infty)$ and for any $\psi=$ $\psi(x) \in S S\left(\mathbb{R}^{N}\right)$ its Fourier transform $\mathcal{F} \varphi$ belongs to $S S\left(\mathbb{R}^{N}\right)$ so that

$$
\left\langle\widehat{\rho}^{\varepsilon}, \varphi \otimes \psi\right\rangle=\left\langle\rho^{\varepsilon}, \mathcal{L} \varphi \otimes \mathcal{F} \psi\right\rangle \rightarrow\langle\eta, \mathcal{L} \varphi \otimes \mathcal{F} \psi\rangle=\langle\widehat{\eta}, \varphi \otimes \psi\rangle
$$

as $\varepsilon \rightarrow 0$. We easily deduce that $\widehat{\rho^{\varepsilon}} \rightarrow \widehat{\eta}$ weakly in $L^{\infty}\left(a, \infty ; L^{2}\left(\mathbb{R}^{N}\right)\right)$ for any $a>0$. Gathering all the convergence results established above, we may pass to the limit in (19) and we get that $\eta$ satisfies

$$
\widehat{\rho_{0}}+\left(-p-\kappa|k|^{\alpha}\right) \widehat{\eta}=0 \quad \text { for a.e. } p>0, k \in \mathbb{R}^{N} \text {. }
$$


Finally the unique solution $\rho$ to the fractional diffusion equation (11), (12), (13) also satisfies

$$
\widehat{\rho_{0}}+\left(-p-\kappa|k|^{\alpha}\right) \widehat{\rho}=0 \quad \text { for a.e. } p>0, k \in \mathbb{R}^{N},
$$

so that $\widehat{\eta}=\widehat{\rho}$ a.e., and then $\eta=\rho$ because the Laplace-Fourier transform is a one-to-one mapping (say in $\mathcal{S}^{\prime}\left([0, \infty) \times \mathbb{R}^{N}\right)$ ). We conclude that $f^{\varepsilon} \rightarrow \rho F$ weakly in $L^{\infty}\left(0, T ; L^{2}\left(\mathbb{R}^{N} \times \mathbb{R}^{N}\right)\right)$ thanks to (17).

\section{Diffusion limit results for general collision oper- ator}

Having exposed the main ideas of the proof in the previous section, we now come back to more general collision operators, and state our main result.

We recall that $L$ now is of the form

$$
\begin{aligned}
L(f) & =\int_{\mathbb{R}^{N}}\left[\sigma\left(v, v^{\prime}\right) f\left(v^{\prime}\right)-\sigma\left(v^{\prime}, v\right) f(v)\right] d v^{\prime} \\
& =K(f)-\nu f
\end{aligned}
$$

with

$$
K(f)=\int_{\mathbb{R}^{N}} \sigma\left(v, v^{\prime}\right) f\left(v^{\prime}\right) d v^{\prime}, \quad \nu(v)=\int_{\mathbb{R}^{N}} \sigma\left(v^{\prime}, v\right) d v^{\prime} .
$$

Such an operator is obviously linear, well-defined as a (possibly) unbounded operator with domain $L^{1}(\nu)$, and closed (in fact it is bounded from $L^{1}(\nu)$ to $L^{1}$ ). It is also straightforwardly conservative:

$$
\int_{\mathbb{R}^{N}} L(h) d v=0 \quad \text { for all } h \in L^{1}(\nu) .
$$

The choice of the cross-section $\sigma\left(v, v^{\prime}\right)$ is crucial. We start with the following structural assumption.

Assumptions (A1) The cross-section $\sigma$ is locally integrable on $\mathbb{R}^{2 N}$, non negative and the collision frequency $\nu$ is locally integrable on $\mathbb{R}^{N}$ and satisfies

$$
\nu(-v)=\nu(v)>0 \quad \text { for all } v \in \mathbb{R}^{N} .
$$

Assumptions (A2) There exists a function $0 \leq F \in L^{1}(\nu)$ such that $|v|^{2} \nu(v)^{-1} F$ is locally integrable and

$$
\nu(v) F(v)=K(F)(v)=\int_{\mathbb{R}^{N}} \sigma\left(v, v^{\prime}\right) F\left(v^{\prime}\right) d v^{\prime},
$$


which means that $F$ is a equilibrium distribution (i.e. $L(F)=0$ ). Furthermore, the function $F$ is symmetric, positive and normalized to 1:

$$
F(-v)=F(v)>0 \text { for all } v \in \mathbb{R}^{N} \quad \text { and } \quad \int_{\mathbb{R}^{N}} F(v) d v=1 .
$$

Note that under classical assumptions on $\sigma$, the existence of an equilibrium function is in fact a consequence of Krein-Rutman's theorem (see [7] for details). A particular case in which this condition is satisfied is when $\sigma$ is such that

$$
\forall v, v^{\prime} \in \mathbb{R}^{N} \quad \sigma\left(v, v^{\prime}\right)=b\left(v, v^{\prime}\right) F(v), \quad b\left(v^{\prime}, v\right)=b\left(v, v^{\prime}\right),
$$

for some $b \in L_{l o c}^{1}\left(\mathbb{R}^{2 N}\right)$. In that case, we say that $\sigma$ satisfies a detailed balanced principle or a micro-reversibility principle, while the more general assumption (22) is called a general balanced principle.

Next, we need to make precise the behavior of $F$ and $\nu$ for large $|v|$. For that purpose, we recall that a slowly varying function is a measurable function $\ell: \mathbb{R}_{+} \rightarrow \mathbb{R}$ such that

$$
\ell(\lambda s) \sim \ell(s) \quad \text { as } s \rightarrow \infty \text { for all } \lambda>0 .
$$

Example of slowly varying functions are positive constants, functions that converge to positive constants, logarithms and iterated logarithms.

In our main result, we assume that $F$ is a regularly varying function of index $-(N+\alpha)$ with $\alpha>0$ and that $\nu$ behaves in the large velocity asymptotic like a power function. More precisely, we make the following assumption:

Assumptions (B1) There exists $\alpha>0$ and a slowly varying function $\ell$ such that

$$
F(v)=F_{0}(v) \ell(|v|)
$$

where $F_{0}$ is such that

$$
|v|^{\alpha+N} F_{0}(v) \longrightarrow \kappa_{0} \in(0, \infty) \text { as }|v| \rightarrow \infty .
$$

Assumptions (B2) There exists $\beta \in \mathbb{R}$ and a positive constant $\nu_{0}$ such that

$$
|v|^{-\beta} \nu(v) \longrightarrow \nu_{0} \quad \text { as }|v| \rightarrow \infty .
$$


Assumptions (B3) Finally we assume that there exists a constant $M$ such that

$$
\int_{\mathbb{R}^{N}} F^{\prime} \frac{\nu}{b} d v^{\prime}+\left(\int_{\mathbb{R}^{N}} \frac{F^{\prime}}{\nu^{\prime}} \frac{b^{2}}{\nu^{2}} d v^{\prime}\right)^{1 / 2} \leq M \quad \text { for all } \quad v \in \mathbb{R}^{N},
$$

with $b=b\left(v, v^{\prime}\right):=\sigma\left(v, v^{\prime}\right) F^{-1}(v)$.

\section{Remarks \& Examples 3.1.}

(i) The constant $\kappa_{0}$ in (25) could actually be a function of the direction $v /|v|$ without any additional difficulties. We will take $\kappa_{0}$ constant in order to keep things simple.

(ii) The condition (B2) implies that the collision frequency satisfies:

$$
\nu_{1}\langle v\rangle^{\beta} \leq \nu(v) \leq \nu_{2}\langle v\rangle^{\beta} \text { for large } v \in \mathbb{R}^{N} .
$$

for some constants $\nu_{1}, \nu_{2} \in(0, \infty)$.

(iii) The conditions (B2) and (B3) are fulfilled for a collision kernel $\sigma$ satisfying the detailed balance principle (23) where $b$ satisfies

$$
b\left(v, v^{\prime}\right)=\langle v\rangle^{\beta}\left\langle v^{\prime}\right\rangle^{\beta} \quad \text { or } \quad b\left(v, v^{\prime}\right)=\left\langle v-v^{\prime}\right\rangle^{\beta}, \quad \beta<\alpha,
$$

since then $\nu(v) \sim\langle v\rangle^{\beta}$ in both cases (for the second example, we refer to the proof of Lemma 6.1 in the appendix where the main arguments of the proof of that statement is presented). Let us notice that such collision kernels satisfy [7, Assumptions (A1), (A2), (A3)] but of course not in general [7, Assumptions (B2), (B3)] except when $\beta>2-\alpha$.

(iv) The conditions (B2) and (B3) are also fulfilled for a (more physical) collision kernel $\sigma$ satisfying the detailed balance principle (23) with

$$
b\left(v, v^{\prime}\right)=\left|v-v^{\prime}\right|^{\beta}
$$

and under the additional restriction $\beta \in(-\min \{\alpha ; N / 2\}, \min \{\alpha ; N)\}$. We refer to Lemma 6.1 in the appendix where the proof of that statement is presented.

(v) Our assumption (B3) is a bit more general than the corresponding assumptions (A3) in [7] since for instance it is fulfilled by the collision kernels of the point (iv) above with $\beta \in(0, \min \{\alpha ; N\})$, while such a collision kernel does not satisfy [7, Assumption (A3)].

We can now state our main theorem: 
Theorem 3.2 (Fractional diffusion limit). Assume that Assumptions (A1A2) and (B1-B2-B3) hold with $\alpha>0$ and $\beta<\min \{\alpha ; 2-\alpha\}$. Define

$$
\gamma:=\frac{\alpha-\beta}{1-\beta}, \quad \text { and } \quad \theta(\varepsilon):=\ell\left(\varepsilon^{-\frac{1}{1-\beta}}\right) \varepsilon^{\gamma} .
$$

It is worth noticing that we have here $\beta<1$ as well $\gamma<2$ for these ranges of the parameters $\alpha$ and $\beta$. Assume furthermore that $f_{0} \in L^{2}\left(F^{-1}\right)$ and let $f^{\varepsilon}$ be the solution of (3), with that choice of $\theta$ and initial data $f_{0}$.

Then, $\left(f^{\varepsilon}\right)$ converges in $L^{\infty}\left(0, T ; L^{2}\left(\mathbb{R}^{N} \times \mathbb{R}^{N}\right)\right)$-weak to a function $\rho(t, x) F(v)$ where $\rho(t, x)$ is the unique solution of the fractional diffusion equation of or$\operatorname{der} \gamma$ :

$$
\begin{array}{ll}
\partial_{t} \rho+\kappa\left(-\Delta_{x}\right)^{\gamma / 2} \rho=0 & \text { in }(0, \infty) \times \mathbb{R}^{N}, \\
\rho(0, .)=\rho_{0} & \text { in } \mathbb{R}^{N},
\end{array}
$$

with $\kappa$ given by

$$
\kappa=\frac{\kappa_{0} \nu_{0}}{1-\beta} \int_{\mathbb{R}^{N}} \frac{w_{1}^{2}}{\nu_{0}^{2}+w_{1}^{2}} \frac{1}{|w|^{N+\gamma}} d w
$$

\section{Remarks 3.3.}

(i) Note that in Theorem 3.2 we always have $\beta<1$ from the assumptions, and the condition $\beta<\min \{\alpha ; 2-\alpha\}$ is equivalent to the condition $\gamma<2$.

(ii) When $\beta=0$ (i.e. $\nu$ bounded below by a positive constant), then we have $\gamma=\alpha$, and we recover a result similar to that of Theorem 1.1 with more general collision operator and equilibrium states. Note in particular that in that case, the addition of a slowly varying part in $F$ has modified the time scale $\theta(\varepsilon)$, but not the limiting equation.

(iii) When $\alpha \in(0,1)$, the function $\beta \in(-\infty, \alpha) \mapsto \gamma(\beta)=(\alpha-\beta) /(1-$ $\beta$ ) is decreasing from 1 to 0 . When $\alpha=1$, it is defined on $(-\infty, 1)$ and identically constant to 1 . When $\alpha>1$, the function $\beta \in(-\infty, 2-\alpha) \mapsto$ $\gamma(\beta)=(\alpha-\beta) /(1-\beta)$ is increasing from 1 to 2 . It is thus always possible to obtain fractional diffusion limit for a kinetic collisional equation with regularly varying equilibrium $F$ of index $-(N+\alpha)$. In particular, fractional diffusion behavior can arise even when $F$ has finite energy $(\alpha>2)$.

The case $\gamma=2$ (which may occur when $\alpha>1$ ) is critical in the sense that even though the second moment may be infinite (and the usual method yield an infinite diffusion coefficient), the asymptotic behavior can still be described by a standard diffusion equation under the appropriate time scale. 
In this case, the exact behavior of the slowly varying function $\ell$ is crucial, as it may determine whether the second moment is finite or infinite. When it is finite, (for instance $\ell(|v|)=(\ln |v|)^{-2}$ ), then the usual technic yields the diffusion equation (5) under the classical time scale $\theta(\varepsilon)=\varepsilon^{2}$. When it is infinite, then the asymptotic behavior is still described by a diffusion equation, but the time scale has to be modified. These two situations are included in the next theorems: the first one corresponds to anomalous diffusive time scales, while the second one corresponds to classical diffusive time scales.

Theorem 3.4 (Classical diffusion limit with anomalous time scale). Assume that Assumptions (A1-A2) and (B1-B2-B3) hold with

$$
\alpha>1 \text { and } \beta=2-\alpha \quad(\text { i.e. } \gamma=2) \text {, }
$$

and $\ell$ such that

$$
\ell(r) \ln (r) \rightarrow+\infty \quad \text { as } r \rightarrow+\infty
$$

(note that this implies in particular that the second moment of $F$ is infinite).

Then define

$$
\theta(\varepsilon)=\varepsilon^{2} \ell\left(\varepsilon^{-\frac{1}{1-\beta}}\right) \ln \left(\varepsilon^{-1}\right) .
$$

Assume furthermore that $f_{0} \in L^{2}\left(F^{-1}\right)$ and let $f^{\varepsilon}$ be the solution of (3), with $\theta(\varepsilon)$ defined as above and initial data $f_{0}$.

Then, $\left(f^{\varepsilon}\right)$ converges in $L^{\infty}\left(0, T ; L^{2}\left(\mathbb{R}^{N} \times \mathbb{R}^{N}\right)\right)$-weak to $\rho F$ where $\rho=$ $\rho(t, x)$ is the unique solution to the standard diffusion equation

$$
\partial_{t} \rho-\kappa \Delta_{x} \rho=0
$$

with $\kappa$ given by

$$
\kappa=\frac{\kappa_{0} \nu_{0}}{(1-\beta)} \lim _{\lambda \rightarrow 0} \frac{1}{\ln \left(\lambda^{-1}\right)} \int_{|w| \geq \lambda} \frac{w_{1}^{2}}{\nu_{0}^{2}+w_{1}^{2}} \frac{1}{|w|^{N+2}} d w .
$$

\section{Remarks 3.5.}

(i) This is a critical case, in which the variance of the equilibrium distribution $F$ (and therefore the classical diffusion coefficient (6)) are infinite, but the asymptotic symbol still is of order 2 . This case is still referred to as anomalous diffusion, even though we recover a standard diffusion equation, because the time scale is not the usual time scale. 
(ii) Proceeding as Section [2, with $\alpha=2, \beta=0$, and $\ell \equiv 1$, we immediately see that if we would take a time scale $\theta(\varepsilon)=\varepsilon^{2}$, then we should expect to find for the diffusion coefficient:

$$
\kappa=\int_{\mathbb{R}^{N}} \frac{w_{1}^{2}}{1+w_{1}^{2}} \frac{\kappa_{0}}{|w|^{N+2}} d w=\infty .
$$

This is why a different time scale has to be considered in this limiting case $\alpha=2$.

Theorem 3.6 (Classical diffusion limit with classical time scale). Assume that Assumptions (A1-A2) hold as well as the following bounds

$$
\int_{\mathbb{R}^{N}}\left(\frac{\nu(v)}{b\left(v, v^{\prime}\right)}+\frac{\left|v^{\prime}\right|^{2}}{\nu\left(v^{\prime}\right)}\right) F^{\prime} d v^{\prime} \leq M \quad \forall v \in \mathbb{R}^{N} .
$$

Assume furthermore that $f_{0} \in L^{2}\left(F^{-1}\right)$ and let $f^{\varepsilon}$ be the solution of (3), with $\theta(\varepsilon)=\varepsilon^{2}$ and initial data $f_{0}$.

Then $\left(f^{\varepsilon}\right)$ converges in $L^{\infty}\left(0, T ; L^{2}\left(\mathbb{R}^{N} \times \mathbb{R}^{N}\right)\right)$-weak and in $L^{2}((0, T) \times$ $\left.\mathbb{R}^{N} \times \mathbb{R}^{N}\right)$ )-strong to a function $\rho F$ where $\rho=\rho(t, x)$ is the unique solution of the standard diffusion equation (5) explained in the introduction (with the same constant).

\section{Remarks 3.7.}

(i) Remark that Asumptions (B1-B2-B3) with any $\beta>2-\alpha$ (i.e. $\gamma>2$ ) obviously implies (33), so that all the values of $\alpha>0, \beta<\min \{1 ; \alpha\}$ are addressed by Theorems 3.2, 3.4 and 3.6. One also sees from these exemples of coefficients that this theorem covers cases where the velocity distribution has infinite variance.

(ii) Theorem 3.6 is (in some direction!) slightly more general than [7, Theorem 1] since for instance [7, Asumption (A3)] implies that b is uniformly bounded from below, whereas Theorem 3.6 applies to the collision kernels of Remarks 85 Examples 3.1 (iv) with $\beta \in(\max \{2-\alpha ; 0\}, \min \{\alpha ; N\})$, while they are not dealt with by [7, Theorem 1].

Figure 1 summarizes a large part of the results presented in the last three theorems.

We now turn to the proofs of Theorems 3.2 and 3.4, presented respectively in Sections 4 and 5. Concerning Theorem 3.6 we shall not write a full proof since it just follows from the proof of [7, Theorem 1] by replacing the proof of the auxiliary result in [7, Proposition 1] by Lemma 4.1 below. 


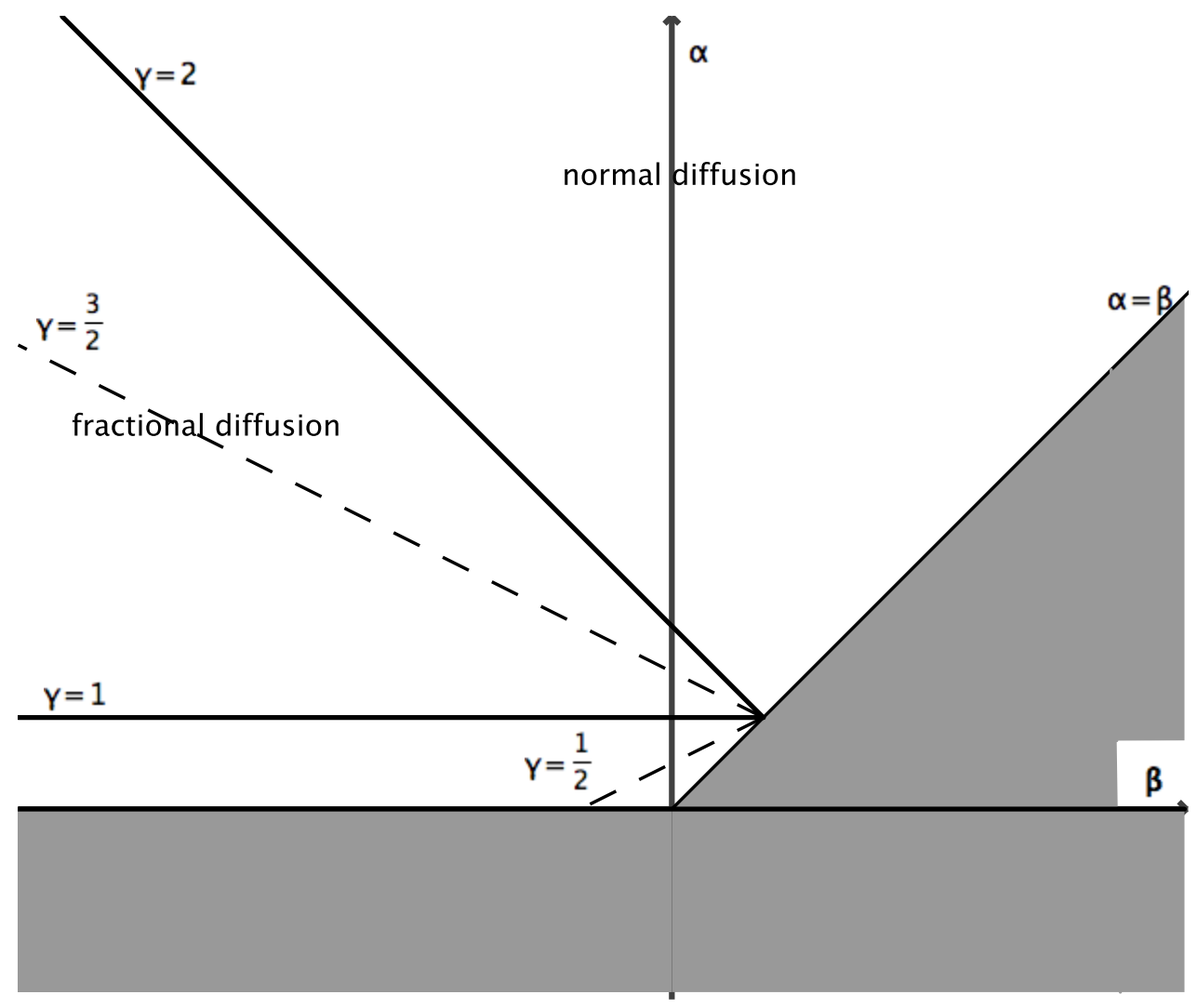

Figure 1: Summary of the main results 


\section{Proof of Theorem 3.2}

The general idea of the proof is the same as that of Theorem 1.1 (see Section 22). The main difference is in the derivation of formulation (19): It is no longer possible to work with the density $\rho^{\varepsilon}$ directly, and we use $K\left(f^{\varepsilon}\right)$ instead leading to the corresponding formulation (39). This leads to another difficulty, since (39) involves an additional term which we have to show is of smaller order. Finally, we will see that the computation of the asymptotic symbol is a little bit more complicated than in Section 2 because of the collision frequency being velocity dependent, and because of the presence of the slowly varying function $\ell$ in $F$ (this is the object of Proposition 4.5, in which we see appearing the importance of the different time scale that takes into account $\ell$ and $\beta$ ).

Throughout this section (and the next one), we denote

$$
\varphi(\varepsilon):=\ell\left(\varepsilon^{-\frac{1}{1-\beta}}\right) .
$$

\subsection{A priori estimates}

The next lemma summarizes the key properties of the collision operator $L$ :

Lemma 4.1. The operator $\frac{1}{\nu} L$ is bounded in $L^{2}\left(\nu F^{-1}\right)$ and satisfies:

$$
\int_{\mathbb{R}^{N}} L(f) \frac{f}{F} d v \leq-\frac{1}{2 M} \int_{\mathbb{R}^{N}}|f-\langle f\rangle F|^{2} \frac{\nu}{F} d v \quad \text { for all } f \in L^{2}\left(\nu F^{-1}\right)
$$

where $\langle f\rangle=\int_{\mathbb{R}^{N}} f(v) d v$ and $M$ is defined in (27).

Proof of Lemma 4.1, We adapt the proof of [7, Proposition 1 \& 2].

To show that $\frac{1}{\nu} L$ is bounded in $L^{2}\left(\nu F^{-1}\right)$, we obviously only have to check that $\frac{1}{\nu} K$ is bounded. Using Cauchy-Schwartz inequality and the fact 
that $K(F)=\nu F$, we get:

$$
\begin{aligned}
\left\|\frac{1}{\nu} K(f)\right\|_{L_{\nu F-1}^{2}}^{2} & =\int \frac{K(f)^{2}}{\nu F} d v \\
& \leq \int \frac{1}{\nu(v) F(v)} \int \sigma\left(v, v^{\prime}\right) F\left(v^{\prime}\right) d v^{\prime} \int \sigma\left(v, v^{\prime}\right) \frac{f\left(v^{\prime}\right)^{2}}{F\left(v^{\prime}\right)} d v^{\prime} d v \\
& \leq \int \frac{1}{\nu(v) F(v)} \nu(v) F(v) \int \sigma\left(v, v^{\prime}\right) \frac{f\left(v^{\prime}\right)^{2}}{F\left(v^{\prime}\right)} d v^{\prime} d v \\
& \leq \iint \sigma\left(v, v^{\prime}\right) \frac{f\left(v^{\prime}\right)^{2}}{F\left(v^{\prime}\right)} d v^{\prime} d v \\
& \leq \int \nu\left(v^{\prime}\right) \frac{f\left(v^{\prime}\right)^{2}}{F\left(v^{\prime}\right)} d v^{\prime}=\|f\|_{L^{2}\left(\nu F^{-1}\right)}^{2}
\end{aligned}
$$

In order to prove (34), we write

$$
\begin{aligned}
\int_{\mathbb{R}^{N}} L(f) \frac{f}{F} d v & =\int_{\mathbb{R}^{N}} \int_{\mathbb{R}^{N}} \sigma\left(v, v^{\prime}\right) f^{\prime} \frac{f}{F} d v d v^{\prime}-\int_{\mathbb{R}^{N}} \nu(v) \frac{f^{2}}{F} d v \\
& =\int_{\mathbb{R}^{N}} \int_{\mathbb{R}^{N}} \sigma\left(v, v^{\prime}\right) F^{\prime} \frac{f^{\prime}}{F^{\prime}} \frac{f}{F} d v d v^{\prime}-\int_{\mathbb{R}^{N}} \nu(v) \frac{f^{2}}{F} d v .
\end{aligned}
$$

Next, we note that the second term in the right hand side can be rewritten

$$
\begin{aligned}
\int_{\mathbb{R}^{N}} \nu(v) \frac{f^{2}}{F} d v & =\int_{\mathbb{R}^{N}} \int_{\mathbb{R}^{N}} \sigma\left(v^{\prime}, v\right) F \frac{f^{2}}{F^{2}} d v d v^{\prime} \\
& =\int_{\mathbb{R}^{N}} \int_{\mathbb{R}^{N}} \sigma\left(v, v^{\prime}\right) F^{\prime} \frac{f^{\prime 2}}{F^{\prime 2}} d v d v
\end{aligned}
$$

as well as (using the fact that $\nu F=K(F)$ )

$$
\begin{aligned}
\int_{\mathbb{R}^{N}} \nu(v) \frac{f^{2}}{F} d v & =\int_{\mathbb{R}^{N}} K(F) \frac{f^{2}}{F^{2}} d v \\
& =\int_{\mathbb{R}^{N}} \int_{\mathbb{R}^{N}} \sigma\left(v, v^{\prime}\right) F^{\prime} \frac{f^{2}}{F^{2}} d v d v .
\end{aligned}
$$

We deduce

$$
\int_{\mathbb{R}^{N}} L(f) \frac{f}{F} d v=-\frac{1}{2} \int_{\mathbb{R}^{N}} \int_{\mathbb{R}^{N}} \sigma\left(v, v^{\prime}\right) F^{\prime}\left[\frac{f^{\prime}}{F^{\prime}}-\frac{f}{F}\right]^{2} d v d v^{\prime} .
$$

Integrating (in the $v^{\prime}$ variable) the identity

$$
f F^{\prime}-f^{\prime} F=\left(\frac{f}{F}-\frac{f^{\prime}}{F^{\prime}}\right) F F^{\prime}
$$


we get

$$
g=\int_{\mathbb{R}^{N}}\left(\frac{f}{F}-\frac{f^{\prime}}{F^{\prime}}\right) F F^{\prime} d v^{\prime}
$$

where $g=f-\langle f\rangle F$. The Cauchy-Schwarz inequality implies

$$
g^{2} \leq\left(\int_{\mathbb{R}^{N}}\left(\frac{f}{F}-\frac{f^{\prime}}{F^{\prime}}\right)^{2} \sigma F^{\prime} d v^{\prime}\right)\left(\int_{\mathbb{R}^{N}} \frac{F^{2}}{\sigma} F^{\prime} d v^{\prime}\right),
$$

so that

$$
\int_{\mathbb{R}^{N}} \frac{g^{2}}{F} \nu d v \leq\left(\sup _{v \in \mathbb{R}^{N}} \nu \int_{\mathbb{R}^{N}} \frac{F}{\sigma} F^{\prime} d v^{\prime}\right)\left(\int_{\mathbb{R}^{N}} \int_{\mathbb{R}^{N}}\left(\frac{f}{F}-\frac{f^{\prime}}{F^{\prime}}\right)^{2} \sigma F^{\prime} d v^{\prime} d v\right) .
$$

Gathering (27), (35) and (36) we obtain (34).

Using Lemma 4.1, we can prove the following estimate on $f^{\varepsilon}$ :

Lemma 4.2. The solution $f^{\varepsilon}$ of (3) is bounded in $L^{\infty}\left(0, \infty ; L^{2}\left(F^{-1}\right)\right)$ uniformly with respect to $\varepsilon$. Furthermore, it satisfies:

$$
f^{\varepsilon}=\rho^{\varepsilon} F(v)+g^{\varepsilon},
$$

where the density $\rho^{\varepsilon}=\int_{\mathbb{R}^{N}} f^{\varepsilon} d v$ and the function $g^{\varepsilon}$ are such that

$$
\left\|\rho^{\varepsilon}\right\|_{L^{\infty}\left(0, \infty, L^{2}\right)} \leq\left\|f_{0}\right\|_{L^{2}\left(F^{-1}\right)}
$$

and

$$
\left\|g^{\varepsilon}\right\|_{L^{2}\left(0, \infty ; L^{2}\left(\nu F^{-1}\right)\right)} \leq C\left\|f_{0}\right\|_{L^{2}\left(F^{-1}\right)} \theta(\varepsilon)^{1 / 2} .
$$

In particular $\rho^{\varepsilon}$ converges $L^{\infty}\left(0, T ; L^{2}\right)$-weak to $\rho$, and $f^{\varepsilon}$ converges $L^{\infty}\left(0, T ; L^{2}\left(F^{-1}\right)\right)$ weak to $f=\rho(t, x) F(v)$.

Proof of Lemma 4.2. Multiplying (3) by $f^{\varepsilon} / F$, we get:

$$
\begin{aligned}
\frac{1}{2} \frac{d}{d t} \int_{\mathbb{R}^{2 N}}\left|f^{\varepsilon}\right|^{2} \frac{1}{F} d x d v & =\frac{1}{\theta(\varepsilon)} \int_{\mathbb{R}^{2 N}} L\left(f^{\varepsilon}\right) \frac{f^{\varepsilon}}{F} \\
& \leq-\frac{1}{2 M \theta(\varepsilon)} \int_{\mathbb{R}^{2 N}}\left|f^{\varepsilon}-\rho^{\varepsilon} F\right|^{2} \frac{\nu}{F} d x d v
\end{aligned}
$$

which gives:

$$
\begin{gathered}
\frac{1}{2} \int_{\mathbb{R}^{2 N}}\left|f^{\varepsilon}(t, x, v)\right|^{2} \frac{1}{F} d x d v+\frac{1}{2 M \theta(\varepsilon)} \int_{0}^{t} \int_{\mathbb{R}^{2 N}}\left|f^{\varepsilon}-\rho^{\varepsilon} F\right|^{2} \frac{\nu}{F} d x d v d s \\
\leq \frac{1}{2} \int_{\mathbb{R}^{2 N}}\left|f_{0}(x, v)\right|^{2} \frac{1}{F} d x d v .
\end{gathered}
$$


This inequality shows that $f^{\varepsilon}$ is bounded in $L^{\infty}\left(0, \infty, L^{2}\left(F^{-1}\right)\right)$. Furthermore, denoting $g^{\varepsilon}=f^{\varepsilon}-\rho^{\varepsilon} F$ we also get

$$
\int_{0}^{t} \int_{\mathbb{R}^{2 N}}\left|g^{\varepsilon}\right|^{2} \frac{\nu}{F} d x d v d s \leq C\left\|f_{0}\right\|_{L^{2}\left(F^{-1}\right)} \theta(\varepsilon) .
$$

Finally, Cauchy-Schwarz inequality implies:

$$
\begin{aligned}
\int_{\mathbb{R}^{N}}\left|\rho^{\varepsilon}\right|^{2} d x & =\int_{\mathbb{R}^{N}}\left|\int_{\mathbb{R}^{N}} f^{\varepsilon} d v\right|^{2} d x \\
& \leq \int_{\mathbb{R}^{2 N}}\left|f^{\varepsilon}\right|^{2} \frac{1}{F} d v \int_{\mathbb{R}^{N}} F d v d x=\int_{\mathbb{R}^{2 N}}\left|f^{\varepsilon}\right|^{2} \frac{1}{F} d v d x
\end{aligned}
$$

\subsection{Another formulation of the rescaled equation}

Proceeding as in Section 2, we denote by $\widehat{f^{\varepsilon}}(p, k, v)$ the Laplace-Fourier transform of $f^{\varepsilon}(t, x, v)$ with respect to $t$ and $x$, defined by

$$
\widehat{f^{\varepsilon}}(p, k, v)=\int_{\mathbb{R}^{N}} \int_{0}^{\infty} e^{-p t} e^{-i k \cdot x} f^{\varepsilon}(t, x, v) d t d x .
$$

We define $\widehat{\rho^{\varepsilon}}(p, k)$ and $\widehat{g^{\varepsilon}}(p, k, v)$ similarly, and we denote by $\widehat{f}_{0}(k, v)$ the Fourier transform of $f_{0}$ with respect to $x$.

Now, taking the Laplace-Fourier transform in (3), it is readily seen that $\widehat{f^{\varepsilon}}$ satisfies

$$
\theta(\varepsilon) p \widehat{f^{\varepsilon}}-\theta(\varepsilon) \widehat{f_{0}}+i v \cdot k \widehat{f^{\varepsilon}}=K\left(\widehat{f^{\varepsilon}}\right)-\nu \widehat{f^{\varepsilon}}
$$

which easily yields

$$
\widehat{f^{\varepsilon}}(v)=\frac{\theta(\varepsilon)}{\nu(v)+\theta(\varepsilon) p+\varepsilon i v \cdot k} \widehat{f}_{0}+\frac{1}{\nu(v)+\theta(\varepsilon) p+\varepsilon i v \cdot k} K\left(\widehat{f^{\varepsilon}}\right) .
$$

Multiplying this equality by $\sigma(w, v)$ and integrating with respect to $v$, we obtain

$$
\begin{aligned}
K\left(\widehat{f^{\varepsilon}}\right)(w)= & \int_{\mathbb{R}^{N}} \frac{\theta(\varepsilon) \sigma(w, v)}{\nu(v)+\theta(\varepsilon) p+\varepsilon i v \cdot k} \widehat{f}_{0}(v) d v \\
& +\int_{\mathbb{R}^{N}} \frac{\sigma(w, v)}{(\nu(v)+\theta(\varepsilon) p+\varepsilon i v \cdot k)} K\left(\widehat{f^{\varepsilon}}\right)(v) d v .
\end{aligned}
$$


Finally, integrating with respect to $w$, we get:

$$
\begin{aligned}
\int_{\mathbb{R}^{N}} K\left(\widehat{f^{\varepsilon}}\right)(v) d v= & \int_{\mathbb{R}^{N}} \frac{\theta(\varepsilon) \nu(v)}{\nu(v)+\theta(\varepsilon) p+\varepsilon i v \cdot k} \widehat{f}_{0}(v) d v \\
& +\int_{\mathbb{R}^{N}} \frac{\nu(v)}{\nu(v)+\theta(\varepsilon) p+\varepsilon i v \cdot k} K\left(\widehat{f^{\varepsilon}}\right)(v) d v,
\end{aligned}
$$

and thus

$$
\begin{aligned}
0= & \int_{\mathbb{R}^{N}} \frac{\nu(v)}{\nu(v)+\theta(\varepsilon) p+\varepsilon i v \cdot k} \widehat{f}_{0}(v) d v \\
& +\frac{1}{\theta(\varepsilon)}\left(\int_{\mathbb{R}^{N}}\left[\frac{\nu(v)}{\nu(v)+\theta(\varepsilon) p+\varepsilon i v \cdot k}-1\right] K\left(\widehat{f^{\varepsilon}}\right)(v) d v\right) .
\end{aligned}
$$

Next, using Lemma 4.2, we write

$$
\widehat{f^{\varepsilon}}=\widehat{\rho^{\varepsilon}} F+\widehat{g^{\varepsilon}}
$$

which leads to (using the fact that $K(F)=\nu F)$ :

$$
K(\widehat{f \varepsilon})=\widehat{\rho^{\varepsilon}} \nu F+K\left(\widehat{g^{\varepsilon}}\right) .
$$

We deduce:

$$
\begin{aligned}
0= & \int_{\mathbb{R}^{N}} \frac{\nu(v)}{\nu(v)+\theta(\varepsilon) p+\varepsilon i v \cdot k} \widehat{f_{0}} d v \\
& +\frac{1}{\theta(\varepsilon)}\left(\int_{\mathbb{R}^{N}}\left[\frac{\nu(v)}{\nu(v)+\theta(\varepsilon) p+\varepsilon i v \cdot k}-1\right] \nu F d v\right) \widehat{\rho^{\varepsilon}} \\
& +\frac{1}{\theta(\varepsilon)}\left(\int_{\mathbb{R}^{N}}\left[\frac{\nu(v)}{\nu(v)+\theta(\varepsilon) p+\varepsilon i v \cdot k}-1\right] K\left(\widehat{g}^{\varepsilon}\right) d v\right) .
\end{aligned}
$$

The rest of the proof consists in passing to the limit $\varepsilon \rightarrow 0$ in (39).

In the next two subsections, we shall show that the last term vanishes in Lemma 4.5 and we study in Lemma 4.3 the limit of the second term

$$
a^{\varepsilon}(p, k):=\frac{1}{\theta(\varepsilon)}\left(\int_{\mathbb{R}^{N}}\left[\frac{\nu(v)}{\nu(v)+\theta(\varepsilon) p+\varepsilon i v \cdot k}-1\right] \nu F d v\right)
$$

as $\varepsilon$ goes to zero. This limit will provide the Fourier-Laplace symbol of the asymptotic equation, and is therefore the cornerstone of the proof.

Let us prove that the first term converges to $\widehat{\rho}_{0}$

$$
\int_{\mathbb{R}^{N}} \frac{\nu(v)}{\nu+\theta(\varepsilon) p+\varepsilon i v \cdot k} \widehat{f}_{0} d v \longrightarrow \rho_{0}, \quad \text { for almost all } k \in \mathbb{R}^{N}, p \in \mathbb{R}_{+}
$$


The assumption $f_{0} \in L^{2}\left(F^{-1}\right)$ implies in particular that $f_{0} \in L_{x}^{2}\left(L_{v}^{1}\right)$. Hence its the Fourier transform $\widehat{f}_{0}$ also belongs to $L_{k}^{2}\left(L_{v}^{1}\right)$ by Parseval equality, which means that $\widehat{f}_{0}$ is integrable in $v$ for almost all $k$. Together with (41), this allows to apply the Lebesgue dominated convergence theorem, which yields, for almost every $k$,

$$
\int_{\mathbb{R}^{N}} \frac{\nu(v)}{\nu+\theta(\varepsilon) p+\varepsilon i v \cdot k} \widehat{f}_{0} d v \longrightarrow \int_{\mathbb{R}^{N}} \widehat{f}_{0} d v=\widehat{\rho}_{0}
$$

which proves (40).

\subsection{Passing to the limit in $a^{\varepsilon}$}

The main goal of this section is the proof of the following proposition:

Lemma 4.3. Recall that $\gamma<2$ and $\theta(\varepsilon)=\varepsilon^{\gamma} \varphi(\varepsilon)$, and let

$$
a^{\varepsilon}(p, k):=\frac{1}{\theta(\varepsilon)} \int_{\mathbb{R}^{N}}\left[\frac{\nu(v)}{\nu+\theta(\varepsilon) p+\varepsilon i v \cdot k}-1\right] \nu(v) F(v) d v .
$$

Then

$$
a^{\varepsilon}(p, k) \longrightarrow-p-\kappa|k|^{\gamma}
$$

when $\varepsilon$ goes to zero, locally uniformly with respect to $p \geq 0$ and $k \in \mathbb{R}^{N}$, with $\kappa$ given by (31). Furthermore, $a^{\varepsilon}$ is locally bounded in $[0, \infty] \times \mathbb{R}^{N}$ uniformly w.r.t. $\varepsilon$ : There exists a constant $C$ such that

$$
\left|a^{\varepsilon}(p, k)\right| \leq|p|+C\left(1+|k|^{2}\right) .
$$

Proof of Lemma 4.3. Observing that

$$
\left|\frac{\nu}{\nu+\theta(\varepsilon) p+\varepsilon i v \cdot k}-1\right| \leq 2
$$

we see that $a^{\varepsilon}$ is well defined for any $\varepsilon>0$. Next, we write

$$
\begin{aligned}
1-\frac{\nu}{\nu+\theta(\varepsilon) p+\varepsilon i v \cdot k}= & \frac{\nu+\theta(\varepsilon) p}{(\nu+\theta(\varepsilon) p)^{2}+(\varepsilon v \cdot k)^{2}} \theta(\varepsilon) p \\
& +\frac{\varepsilon^{2}(v \cdot k)^{2}}{(\nu+\theta(\varepsilon) p)^{2}+(\varepsilon v \cdot k)^{2}} \\
& +\frac{\varepsilon \nu i v \cdot k}{(\nu+\theta(\varepsilon) p)^{2}+(\varepsilon v \cdot k)^{2}} .
\end{aligned}
$$


Using the fact that $F(-v)=F(v)$ and $\nu(-v)=\nu(v)$, we deduce

$$
\begin{aligned}
a^{\varepsilon}(p, k)= & -p \int_{\mathbb{R}^{N}} \frac{\nu(v)+\theta(\varepsilon) p}{(\nu(v)+\theta(\varepsilon) p)^{2}+\varepsilon^{2}(v \cdot k)^{2}} \nu(v) F(v) d v \\
& -\frac{1}{\theta(\varepsilon)} \int_{\mathbb{R}^{N}} \frac{(\varepsilon v \cdot k)^{2}}{(\nu(v)+\theta(\varepsilon) p)^{2}+(\varepsilon v \cdot k)^{2}} \nu(v) F(v) d v .
\end{aligned}
$$

The dominated convergence theorem immediately implies that the first term in the right hand side converges to $-p \int_{\mathbb{R}^{N}} F(v) d v=-p$ as $\varepsilon$ goes to zero. Furthermore, that term is clearly bounded (in absolute value) by $|p|$. So it only remains to show that (and here we recall that $\theta(\varepsilon):=\varepsilon^{\gamma} \varphi(\varepsilon)$ )

$$
d^{\varepsilon}(p, k):=\frac{1}{\varepsilon^{\gamma} \varphi(\varepsilon)} \int_{\mathbb{R}^{N}} \frac{(\varepsilon v \cdot k)^{2}}{\left(\nu+\varepsilon^{\gamma} \varphi(\varepsilon) p\right)^{2}+(\varepsilon v \cdot k)^{2}} \nu F d v
$$

converges to $\kappa|k|^{\gamma}$ and is locally bounded when $\varepsilon$ goes to zero.

For some $M>0$, we write

$$
d^{\varepsilon}(p, k)=d_{1}^{\varepsilon}(p, k)+d_{2}^{\varepsilon}(p, k)
$$

where

$$
\begin{aligned}
d_{1}^{\varepsilon}(p, k) & =\frac{1}{\varepsilon^{\gamma} \varphi(\varepsilon)} \int_{|v| \leq M} \frac{(\varepsilon v \cdot k)^{2}}{\left(\nu(v)+\varepsilon^{\gamma} \varphi(\varepsilon) p\right)^{2}+(\varepsilon v \cdot k)^{2}} \nu(v) F(v) d v \\
& \leq \varepsilon^{2-\gamma} \varphi(\varepsilon)^{-1} \int_{|v| \leq M} \nu(v)^{-1}|v \cdot k|^{2} F(v) d v \\
& \leq|k|^{2} \varepsilon^{2-\gamma} \varphi(\varepsilon)^{-1} \int_{|v| \leq M}|v|^{2} \nu(v)^{-1} F(v) d v \\
& \leq C|k|^{2} \varepsilon^{2-\gamma} \varphi(\varepsilon)^{-1}
\end{aligned}
$$

(using the assumption (A2) that $|v|^{2} \nu(v)^{-1} F$ is locally integrable) and

$$
d_{2}^{\varepsilon}(p, k)=\frac{1}{\varepsilon^{\gamma} \varphi(\varepsilon)} \int_{|v| \geq M} \frac{(\varepsilon v \cdot k)^{2}}{\left(\nu(v)+\varepsilon^{\gamma} \varphi(\varepsilon) p\right)^{2}+(\varepsilon v \cdot k)^{2}} \nu(v) F(v) d v .
$$

It is readily seen (using (46) and the fact that $\gamma<2$ ) that

$$
d_{1}^{\varepsilon}(p, k) \longrightarrow 0 \text { as } \varepsilon \rightarrow 0 .
$$

Furthermore, $d_{1}^{\varepsilon}$ is bounded, for $\varepsilon$ small enough by $C|k|^{2}$. So we only need to evaluate the limit of $d_{2}^{\varepsilon}(p, k)$. For that purpose, we first rewrite $d_{2}^{\varepsilon}$ as follows:

$d_{2}^{\varepsilon}(p, k)=\frac{1}{\varepsilon^{\gamma} \varphi(\varepsilon)} \int_{|v| \geq M} \frac{\left(|v|^{-\beta} \varepsilon v \cdot k\right)^{2}}{\left(\widetilde{\nu}(v)+|v|^{-\beta} \varepsilon^{\gamma} \varphi(\varepsilon) p\right)^{2}+\left(|v|^{-\beta} \varepsilon v \cdot k\right)^{2}} \frac{\widetilde{\nu}(v) \widetilde{F}_{0}(v) \ell(|v|)}{|v|^{N+\alpha-\beta}} d v$. 
where

$$
\widetilde{\nu}(v)=|v|^{-\beta} \nu(v) \quad \text { and } \quad \widetilde{F}_{0}(v)=|v|^{N+\alpha} F_{0}(v) .
$$

Note that Assumptions (B1-B2-B3) (see also (28)) imply that $\widetilde{\nu}$ and $\widetilde{F}_{0}$ are uniformly bounded from above and below for $|v| \geq M$ for a suitable $M>0$, and that

$$
\lim _{|v| \rightarrow \infty} \widetilde{\nu}(v)=\nu_{0} \quad \text { and } \quad \lim _{|v| \rightarrow \infty} \widetilde{F}_{0}(v)=\kappa_{0} .
$$

We now do the change of variable

$$
w=\varepsilon|k||v|^{-\beta} v,
$$

for which we have:

$$
|v|=\frac{|w|^{\frac{1}{1-\beta}}}{(\varepsilon|k|)^{\frac{1}{1-\beta}}}, \quad v=\frac{w}{|w|^{\frac{-\beta}{1-\beta}}(\varepsilon|k|)^{\frac{1}{1-\beta}}}, \quad \text { and } \quad d v=\frac{(1-\beta)^{-1}}{(\varepsilon|k|)^{\frac{N}{1-\beta}}|w|^{\frac{-\beta N}{1-\beta}}} d w .
$$

We obtain (with $e=k /|k|$ ):

$$
\begin{aligned}
& d_{2}^{\varepsilon}(p, k)= \\
& =\frac{(1-\beta)^{-1}}{\varepsilon^{\gamma} \varphi(\varepsilon)} \int_{|w| \geq M^{1-\beta} \varepsilon|k|} \frac{(w \cdot e)^{2}}{\left(\widetilde{\nu}^{\varepsilon}(w)+|w|^{\frac{-\beta}{1-\beta}}(\varepsilon|k|)^{\frac{\beta}{1-\beta}} \varepsilon^{\gamma} \varphi(\varepsilon) p\right)^{2}+(w \cdot e)^{2}} \\
& \quad \times \frac{\widetilde{\nu}^{\varepsilon}(w) \widetilde{F}_{0}^{\varepsilon}(w)}{|w|^{\frac{N+\alpha-\beta}{1-\beta}}}(\varepsilon|k|)^{\frac{N+\alpha-\beta}{1-\beta}} \ell\left(\frac{|w|^{\frac{1}{1-\beta}}}{(\varepsilon|k|)^{\frac{1}{1-\beta}}}\right)(\varepsilon|k|)^{-\frac{N}{1-\beta}}|w|^{-\frac{N-\beta}{1-\beta}} d w .
\end{aligned}
$$

where

$$
\widetilde{\nu}^{\varepsilon}(w)=\widetilde{\nu}\left(\frac{w}{|w|^{\frac{-\beta}{1-\beta}}(\varepsilon|k|)^{\frac{1}{1-\beta}}}\right)
$$

and

$$
\widetilde{F}_{0}^{\varepsilon}(w)=\widetilde{F}_{0}\left(\frac{w}{|w|^{\frac{-\beta}{1-\beta}}(\varepsilon|k|)^{\frac{1}{1-\beta}}}\right)
$$

(we dropped the dependence in $k$ in $\widetilde{\nu}^{\varepsilon}$ and $\widetilde{F}_{0}^{\varepsilon}$ to keep notation simpler). The definition of $\gamma$ thus gives:

$$
\begin{aligned}
& d_{2}^{\varepsilon}(p, k)= \\
& =(1-\beta)^{-1}|k|^{\gamma} \int_{|w| \geq M^{1-\beta} \varepsilon|k|} \frac{(w \cdot e)^{2}}{\left(\widetilde{\nu}^{\varepsilon}(w)+|w|^{\frac{-\beta}{1-\beta}}|k|^{\frac{\beta}{1-\beta}} \varphi(\varepsilon) \varepsilon^{\frac{\alpha}{1-\beta}} p\right)^{2}+(w \cdot e)^{2}} \\
& \quad \times \frac{\widetilde{\nu}^{\varepsilon}(w) \widetilde{F}_{0}^{\varepsilon}(w)}{|w|^{N+\gamma}} \ell\left(\frac{|w|^{\frac{1}{1-\beta}}}{(\varepsilon|k|)^{\frac{1}{1-\beta}}}\right) \frac{1}{\varphi(\varepsilon)} d w .
\end{aligned}
$$


Finally, we note that for any $w$ and $k$, we have (note that $\beta<1$ )

$$
\begin{aligned}
& \lim _{\varepsilon \rightarrow 0} \widetilde{\nu}^{\varepsilon}(w)=\nu_{0} \\
& \lim _{\varepsilon \rightarrow 0} \widetilde{F}_{0}^{\varepsilon}(w)=\kappa_{0}
\end{aligned}
$$

and

$$
\lim _{\varepsilon \rightarrow 0} \frac{1}{\varphi(\varepsilon)} \ell\left(\frac{|w|^{\frac{1}{1-\beta}}}{(\varepsilon|k|)^{\frac{1}{1-\beta}}}\right)=\lim _{\varepsilon \rightarrow 0} \frac{1}{\ell\left(\varepsilon^{-\frac{1}{1-\beta}}\right)} \ell\left(\frac{|w|^{\frac{1}{1-\beta}}}{(\varepsilon|k|)^{\frac{1}{1-\beta}}}\right)=1 .
$$

Thus, the integrand in (45) converges pointwise to

$$
\frac{(w \cdot e)^{2}}{\nu_{0}^{2}+(w \cdot e)^{2}} \frac{\nu_{0} \kappa_{0}}{|w|^{N+\gamma}}
$$

Furthermore, it is bounded by

$$
C \frac{(w \cdot e)^{2}}{\nu_{1}^{2}+(w \cdot e)^{2}} \frac{1}{|w|^{N+\gamma}}\left(1+\left(\frac{|w|}{|k|}\right)^{\delta}\right)
$$

thanks to the following lemma and an appropriate choice of $M$, see the following lemma (extracted from [3, Theorem 1.5.6]):

Lemma 4.4 (Potter's Theorem). For all $\delta>0$ there exists some constants $C, M>0$ such that

$$
\left|\frac{\ell(\lambda s)}{\ell(s)}\right| \leq C\left(1+|\lambda|^{\delta}\right) \quad \text { for all } s \geq M \text { and } \lambda \geq M / s .
$$

Moreover for any $\zeta>0$ :

$$
s^{\zeta} \ell(s) \longrightarrow \infty \quad \text { and } \quad s^{-\zeta} \ell(s) \longrightarrow 0 \quad \text { as } \quad s \rightarrow \infty .
$$

Choosing $0<\delta \leq \gamma / 2$ (and thus fixing a corresponding constant $M$ ), we deduce that

$$
\left|d_{2}^{\varepsilon}(p, k)\right| \leq C|k|^{\gamma}\left(1+|k|^{-\delta}\right)
$$

and by Lebesgue's dominated convergence theorem we get:

$$
d_{2}^{\varepsilon}(p, k) \longrightarrow(1-\beta)^{-1}|k|^{\gamma} \int_{\mathbb{R}^{N}} \frac{(w \cdot e)^{2}}{\nu_{0}^{2}+(w \cdot e)^{2}} \frac{\nu_{0} \kappa_{0}}{|w|^{N+\gamma}} d w=\kappa|k|^{\gamma},
$$

which concludes the proof. $\square$ 


\subsection{The remaining term}

We will also need the following technical lemma to pass to the limit in the first and last term in (39). Note that we directly include in this lemma the limit case $\gamma=2$ since we shall need it this same technical lemma in this limit case for the proof of Theorem 3.4 .

Lemma 4.5. Assume (A1-A2) and (B1-B2-B3) with $\alpha>0, \beta<\alpha$ and $\beta \leq 2-\alpha$.

Then

$$
\int_{\mathbb{R}^{N}}\left|\frac{\nu(v)}{\nu+\theta(\varepsilon) p+\varepsilon i v \cdot k}-1\right| \nu(v) F(v) d v \leq C(p, k) \varepsilon^{\min \{\gamma / 2+\eta ; 1\}}
$$

for some $\eta>0$, all $k \in \mathbb{R}^{N}, p \in \mathbb{R}_{+}$, and $C(p, k) \in L_{\text {loc }}^{\infty}\left(\mathbb{R}_{+} \times \mathbb{R}^{N}\right)$.

Remarks 4.6. Note that the assumptions of Lemma 4.5 imply that $0<\gamma \leq$ 2 , and therefore $\gamma=2$ is included in these assumptions, except when $\alpha=1$. In other words we exclude the case $\alpha=\beta=1$ and hence when $\gamma=2$ we always suppose $\alpha>1$.

\section{Proof of Lemma 4.5.}

To prove (47), we split the integral into three part parts:

$$
\int_{\mathbb{R}^{N}}\left|\frac{\nu(v)}{\nu+\theta(\varepsilon) p+\varepsilon i v \cdot k}-1\right| \nu(v) F(v) d v=c_{+}^{\varepsilon}(p, k)+c_{-}^{\varepsilon}(p, k)
$$

where

$$
c_{ \pm}^{\varepsilon}(p, k):=\int_{\left\{|v|^{ \pm 1} \leq \varepsilon^{\frac{\mp 1}{1-\beta}}\right\}}\left|\frac{\nu(v)}{\nu+\theta(\varepsilon) p+\varepsilon i v \cdot k}-1\right| \nu(v) F(v) d v
$$

(note that this splitting is well-defined since $\beta \neq 1$ in the assumptions).

In order to get a bound for $c_{+}^{\varepsilon}$, we note that (42) implies:

$$
\left|\frac{\nu}{\nu+\theta(\varepsilon) p+\varepsilon i v \cdot k}-1\right| \leq \nu^{-1} \theta(\varepsilon)|p|+\varepsilon^{2} \nu^{-2}(v \cdot k)^{2}+\varepsilon \nu^{-1}|v \cdot k|
$$

and thus

$$
\begin{aligned}
c_{+}^{\varepsilon}(p, k) \leq & \theta(\varepsilon)|p| \int_{\mathbb{R}^{N}} F(v) d v \\
& +(\varepsilon|k|)^{2} \int_{\left\{|v| \leq \varepsilon^{\frac{-1}{1-\beta}}\right\}}|v|^{2} \nu^{-1}(v) F(v) d v \\
& \left.+\varepsilon|k| \int_{\left\{|v| \leq \varepsilon^{\frac{-1}{1-\beta}}\right.}\right\}^{|v| F(v) d v .}
\end{aligned}
$$


We write

$$
c_{+}^{\varepsilon}(p, k) \leq \theta(\varepsilon)|p|+(\varepsilon|k|)^{2} c_{+, 2}^{\varepsilon}(p, k)+\varepsilon|k| c_{+, 3}^{\varepsilon}(p, k) .
$$

The computation of $c_{+, 2}^{\varepsilon}(p, k)$ requires the computation of the following integral:

$$
\int_{\left\{|v| \leq \varepsilon^{\frac{-1}{1-\beta}}\right\}}|v|^{2} \nu^{-1}(v) F(v) d v
$$

which we estimate using the assumption (A2) that $|v|^{2} \nu(v)^{-1} F$ is locally integrable, and the fact that $F(v) \leq C_{1} \ell(v)|v|^{-N-\alpha}$ and $\nu(v) \geq C_{2}|v|^{\beta}$ for $|v| \geq M$. When $\beta \neq 2-\alpha$, it yields

$$
c_{+, 2}^{\varepsilon} \leq C\left(1+\varepsilon^{\gamma-2-\delta}\right)
$$

for some $\delta$ as small as we want, using the Potter's lemma 4.4 to estimate $\ell$. In the case when $\beta=2-\alpha$, it yields

$$
c_{+, 2}^{\varepsilon} \leq C\left(1+\Xi\left(\varepsilon^{-1 /(1-\beta)}\right)\right)
$$

with

$$
\Xi(z):=\int_{M}^{z} \frac{\ell(r)}{r} d r \text { if } z \geq M, \quad \Xi(z):=0 \text { if } z \leq M .
$$

In both cases this yields, by fixing $\delta=\gamma / 2$, using the trivial estimate

$$
z^{-\delta} \Xi(z) \underset{z \rightarrow+\infty}{\longrightarrow} 0
$$

for any $\delta>0$, and keeping only higher order terms, we obtain the following (non optimal) bound:

$$
(\varepsilon|k|)^{2} c_{+, 2}^{\varepsilon}(p, k) \leq C \varepsilon^{\gamma / 2+\eta}
$$

for some $\eta>0$.

Similarly we found

$$
c_{+, 3}^{\varepsilon} \leq C\left(1+\varepsilon^{\gamma-1-\delta}\right) \text { if } \alpha \neq 1, \quad c_{+, 3}^{\varepsilon} \leq C\left(1+\Xi\left(\varepsilon^{-1 /(1-\beta)}\right)\right) \text { if } \alpha=1
$$

for some $\delta$ as small as we want.

Using the same arguments as before (and recalling that when $\alpha=1$, $\gamma=2$ is not allowed since $\beta<\alpha$ ), we get

$$
(\varepsilon|k|) c_{+, 3}^{\varepsilon}(p, k) \leq C(\varepsilon|k|)^{\min \{\gamma / 2+\eta ; 1\}}
$$


for some $\eta>0$.

Finally, in order to get a bound on $c_{-}^{\varepsilon}$, we do the change of variable $w=\varepsilon^{\frac{1}{1-\beta}} v$ which leads to

$$
\begin{aligned}
& c_{-}^{\varepsilon}(p, k) \leq \int\left\{|v| \geq \varepsilon^{\frac{-1}{1-\beta}}\right\}^{2 \nu(v) F(v) d v} \\
& \leq 2 \varepsilon^{\frac{\alpha-\beta}{1-\beta}} \int_{|w| \geq 1} \frac{\widetilde{\nu^{\varepsilon}}(w) \widetilde{F}_{0}^{\varepsilon}(w)}{|w|^{N+\alpha-\beta}} \ell\left(|w| \varepsilon^{\frac{-1}{1-\beta}}\right) d w \\
& \leq C \varepsilon^{\gamma} \varphi(\varepsilon) \int_{|w| \geq 1}|w|^{-N-\alpha+\beta}\left(1+|w|^{\delta}\right) d w \\
& \leq C^{\prime} \varepsilon^{\gamma} \varphi(\varepsilon)
\end{aligned}
$$

where we used Potter's lemma 4.4 for some $\delta>0$.

Collecting all the terms, and keeping only higher order terms, we find easily (47).

Finally we show that the last term in (39) goes to zero in $\mathcal{D}^{\prime}\left([0, \infty] \times \mathbb{R}^{N}\right)$ : First, we note that for any $g \in L^{2}\left(\nu F^{-1}\right)$, we have by Cauchy-Schwarz inequality and assumption (27)

$$
\begin{aligned}
|K(g)| & \leq \int_{\mathbb{R}^{N}} \sigma\left(v, v^{\prime}\right)\left|g\left(v^{\prime}\right)\right| d v^{\prime} \\
& \leq\left(\int_{\mathbb{R}^{N}} \sigma^{2} \frac{F^{\prime}}{\nu^{\prime}} d v^{\prime}\right)^{1 / 2}\left(\int_{\mathbb{R}^{N}} \frac{\left|g\left(v^{\prime}\right)\right|^{2}}{F\left(v^{\prime}\right)} \nu^{\prime} d v^{\prime}\right)^{1 / 2} \\
& \leq M \nu F\|g\|_{L^{2}\left(\nu F^{-1}\right)}
\end{aligned}
$$

Next, using Lemma 4.5, we deduce that

$$
\begin{aligned}
\mid \frac{1}{\theta(\varepsilon)} & \int_{\mathbb{R}^{N}}\left[\frac{\nu(v)}{\nu(v)+\theta(\varepsilon) p+\varepsilon i v \cdot k}-1\right] K\left(\widehat{g^{\varepsilon}}\right)(v) d v \mid \\
& \leq \frac{C}{\theta(\varepsilon)}\left(\int_{\mathbb{R}^{N}}\left|\frac{\nu(v)}{\nu(v)+\theta(\varepsilon) p+\varepsilon i v \cdot k}-1\right| \nu(v) F(v) d v\right)\left\|\widehat{g}^{\varepsilon}\right\|_{L^{2}\left(\nu F^{-1}\right)} \\
& \leq \frac{C(p, k)}{\theta(\varepsilon)} \varepsilon^{\gamma / 2+\eta}\left\|\widehat{g^{\varepsilon}}\right\|_{L^{2}\left(\nu F^{-1}\right)}
\end{aligned}
$$

Note that here since $\gamma<2$, one can find $\eta>0$ such that $\gamma / 2+\eta<1$, and therefore the estimate (47) in Lemma 4.5 yields a bound from the below of the form $C \varepsilon^{\gamma / 2+\eta}$. 
Last, recalling inequality (38), we have for any test function $\phi \in \mathcal{D}((0, \infty) \times$ $\left.\mathbb{R}^{N}\right)$ :

$$
\begin{aligned}
& \mid \int_{0}^{\infty} \int_{\mathbb{R}^{N}} \phi(p, k) \frac{1}{\theta(\varepsilon)} \int_{\mathbb{R}^{N}}\left[\frac{\nu(v)}{\nu(v)+\theta(\varepsilon) p+\varepsilon i v \cdot k}-1\right] K\left(\widehat{g^{\varepsilon}}\right)(v) d v d k d p \mid \\
& \leq \frac{C \varepsilon^{\gamma / 2+\eta}}{\theta(\varepsilon)}\left(\int_{0}^{\infty}\left(\int_{\mathbb{R}^{N}} C(p, k)^{2} \phi(p, k)^{2} d k\right)^{1 / 2} d p\right) \\
& \times\left\|\widehat{g^{\varepsilon}}\right\|_{L^{\infty}\left((a, \infty) ; L^{2}\left(\nu F^{-1}\right)\right)} \\
& \leq \frac{C(\phi) \varepsilon^{\gamma / 2+\eta}}{\theta(\varepsilon)}\left\|g^{\varepsilon}\right\|_{L^{2}\left((0, \infty) ; L^{2}\left(\nu F^{-1}\right)\right)} \\
& \leq C(\phi) \varepsilon^{\eta} \varphi(\varepsilon)^{-1}
\end{aligned}
$$

which goes to zero thanks to (46) and the fact that $\eta>0$.

\subsection{Conclusion}

We now have all the tools to pass to the limit $\varepsilon \rightarrow 0$ in (39). The first part of Lemma 4.5 implies that the first term converges to $\widehat{\rho_{0}}(k)$ locally uniformly with respect to $k$ and $p$, while the second term can be rewritten as $a^{\varepsilon}(p, k) \widehat{\rho^{\varepsilon}}$ which converges, using Lemma 4.3, in $\mathcal{D}^{\prime}\left((0, \infty) \times \mathbb{R}^{N}\right)$ to $\left(-p-\kappa|k|^{\gamma}\right) \widehat{\rho}$.

The remaining term goes to 0 thanks the the second part of Lemma 4.5 and the preceeding subsection.

Hence we can pass to the limit in (39) and recover (21). The proof of Theorem 3.2 can then be completed as the proof of Theorem 1.1 ,

\section{Proof of Theorem 3.4}

The proof of Theorem 3.6 is very similar to the proof of Theorem 3.2 . We recall the main steps for the reader's sake.

We recall that we define the following time scale in the critical case:

$$
\theta(\varepsilon)=\varepsilon^{2} \ell\left(\varepsilon^{-\frac{1}{1-\beta}}\right) \ln \left(\varepsilon^{-1}\right) .
$$

and we shall use the same notation as in the previous section:

$$
\varphi(\varepsilon)=\ell\left(\varepsilon^{-\frac{1}{1-\beta}}\right) .
$$


The starting point is again the equality (39). As in the previous section, we see that if we take that the first term converges to $\rho_{0}$ when $\varepsilon$ goes to zero.

Proceeding as in Section 4.4 we can also show (using Lemma 4.5 with $\gamma=2)$ that the last term goes to zero:

$$
\begin{aligned}
\mid \frac{1}{\theta(\varepsilon)} & \int_{\mathbb{R}^{N}}\left[\frac{\nu(v)}{\nu(v)+\theta(\varepsilon) p+\varepsilon i v \cdot k}-1\right] K\left(\widehat{g^{\varepsilon}}\right)(v) d v \mid \\
& \leq \frac{C}{\theta(\varepsilon)}\left(\int_{\mathbb{R}^{N}}\left|\frac{\nu(v)}{\nu(v)+\theta(\varepsilon) p+\varepsilon i v \cdot k}-1\right| \nu(v) F(v) d v\right)\left\|\widehat{g^{\varepsilon}}\right\|_{L^{2}\left(\nu F^{-1}\right)} \\
& \leq \frac{C(p, k)}{\theta(\varepsilon)} \varepsilon\left\|\widehat{g^{\varepsilon}}\right\|_{L^{2}\left(\nu F^{-1}\right)} .
\end{aligned}
$$

Note that here since $\gamma=2$, the estimate (47) in Lemma 4.5 yields a bound from the below of the form $C \varepsilon$. $\left.\mathbb{R}^{N}\right)$ :

Last, recalling inequality (38), we have for any test function $\phi \in \mathcal{D}((0, \infty) \times$

$$
\begin{aligned}
& \left|\int_{0}^{\infty} \quad \int_{\mathbb{R}^{N}} \phi(p, k) \frac{1}{\theta(\varepsilon)} \int_{\mathbb{R}^{N}}\left[\frac{\nu(v)}{\nu(v)+\theta(\varepsilon) p+\varepsilon i v \cdot k}-1\right] K\left(\widehat{g^{\varepsilon}}\right)(v) d v d k d p\right| \\
& \quad \leq \frac{C \varepsilon}{\theta(\varepsilon)}\left(\int_{0}^{\infty}\left(\int_{\mathbb{R}^{N}} C(p, k)^{2} \phi(p, k)^{2} d k\right)^{1 / 2} d p\right)\left\|\widehat{g}^{\varepsilon}\right\|_{L^{\infty}\left((a, \infty) ; L^{2}\left(\nu F^{-1}\right)\right)} \\
& \quad \leq \frac{C(\phi) \varepsilon}{\theta(\varepsilon)}\left\|g^{\varepsilon}\right\|_{L^{2}\left((0, \infty) ; L^{2}\left(\nu F^{-1}\right)\right)} \\
& \quad \leq \frac{C(\phi)}{\varphi(\varepsilon) \ln \left(\varepsilon^{-1}\right)}
\end{aligned}
$$

which goes to zero thanks to assumption (32) in Theorem 3.4 $\ell(r) \ln r \rightarrow$ $+\infty$ as $r \rightarrow+\infty$ indeed immediately implies that $\ell\left(\varepsilon^{-1 /(1-\beta)}\right) \ln \left(\varepsilon^{-1}\right) \rightarrow$ $+\infty$ as $\varepsilon \rightarrow 0$ (recall that here $1-\beta=\alpha-1>0$ ).

Finally, we are left with the task of determining the limit of the symbol $a^{\varepsilon}$ in (43).

It has already been proved that the first term in the right hand side of (43) is bounded (uniformly in $\varepsilon$ ) by $|p|$ and converges to $-p \int_{\mathbb{R}^{N}} F(v) d v=$ $-p$ as $\varepsilon$ goes to zero.

So it only remains to show that

$$
d^{\varepsilon}(p, k):=\frac{1}{\theta(\varepsilon)} \int_{\mathbb{R}^{N}} \frac{(\varepsilon v \cdot k)^{2}}{(\nu+\theta(\varepsilon) p)^{2}+(\varepsilon v \cdot k)^{2}} \nu(v) F(v) d v
$$

converges to $\kappa|k|^{2}$ and is locally bounded when $\varepsilon$ goes to zero. 
For some $M>0$, we write

$$
d^{\varepsilon}(p, k)=d_{1}^{\varepsilon}(p, k)+d_{2}^{\varepsilon}(p, k),
$$

where

$$
\begin{aligned}
d_{1}^{\varepsilon}(p, k) & =\frac{1}{\theta(\varepsilon)} \int_{|v| \leq M} \frac{(\varepsilon v \cdot k)^{2}}{(\nu(v)+\theta(\varepsilon) p)^{2}+(\varepsilon v \cdot k)^{2}} \nu(v) F(v) d v \\
& \leq \frac{1}{\varphi(\varepsilon) \ln \left(\varepsilon^{-1}\right)} \int_{|v| \leq M} \nu(v)^{-1}|v \cdot k|^{2} F(v) d v \\
& \leq \frac{|k|^{2}}{\varphi(\varepsilon) \ln \left(\varepsilon^{-1}\right)} \int_{|v| \leq M}|v|^{2} \nu(v)^{-1} F(v) d v \\
& \leq \frac{C|k|^{2}}{\varphi(\varepsilon) \ln \left(\varepsilon^{-1}\right)}
\end{aligned}
$$

(using the assumption (A2) that $|v|^{2} \nu(v)^{-1} F$ is locally integrable) and

$$
d_{2}^{\varepsilon}(p, k)=\frac{1}{\theta(\varepsilon)} \int_{|v| \geq M} \frac{(\varepsilon v \cdot k)^{2}}{(\nu(v)+\theta(\varepsilon) p)^{2}+(\varepsilon v \cdot k)^{2}} \nu(v) F(v) d v .
$$

Concerning $d_{1}^{\varepsilon}(p, k)$, as above we deduce from the assumption (32) on $\ell$ that

$$
\varphi(\varepsilon) \ln \left(\varepsilon^{-1}\right) \rightarrow+\infty \quad \text { as } \varepsilon \rightarrow 0,
$$

and therefore $d_{1}^{\varepsilon}(p, k)$ goes to 0 as $\varepsilon$ goes to 0 . Moreover it is clearly bounded, for $\varepsilon$ small enough, by some $C|k|^{2}$.

Now it remains to evaluate the limit of $d_{2}^{\varepsilon}(p, k)$. For that purpose, we first rewrite $d_{2}^{\varepsilon}$ as follows:

$d_{2}^{\varepsilon}(p, k)=\frac{1}{\theta(\varepsilon)} \int_{|v| \geq M} \frac{\left(|v|^{-\beta} \varepsilon v \cdot k\right)^{2}}{\left(\widetilde{\nu}(v)+|v|^{-\beta} \theta(\varepsilon) p\right)^{2}+\left(|v|^{-\beta} \varepsilon v \cdot k\right)^{2}} \frac{\widetilde{\nu}(v) \widetilde{F}_{0}(v)}{|v|^{N+\alpha-\beta}} \ell(|v|) d v$.

where

$$
\widetilde{\nu}(v)=|v|^{-\beta} \nu(v) \quad \text { and } \quad \widetilde{F}_{0}(v)=|v|^{N+\alpha} F_{0}(v) .
$$

Note that Assumptions (B1-B2-B3) (see also (28)) imply that $\widetilde{\nu}$ and $\widetilde{F}_{0}$ are uniformly bounded from above and below for $|v| \geq M$ and that

$$
\lim _{|v| \rightarrow \infty} \widetilde{\nu}(v)=\nu_{0} \quad \text { and } \quad \lim _{|v| \rightarrow \infty} \widetilde{F}_{0}(v)=\kappa_{0} .
$$


We do again the change of variable $w=\varepsilon|k||v|^{-\beta} v$ (recall again that $\beta \neq 1$ from the assumptions), and we obtain (with $e=k /|k|)$ :

$$
\begin{aligned}
& d_{2}^{\varepsilon}(p, k)= \\
& =\frac{(1-\beta)^{-1}}{\theta(\varepsilon)} \int_{|w| \geq M^{1-\beta} \varepsilon|k|} \frac{(w \cdot e)^{2}}{\left(\widetilde{\nu}^{\varepsilon}(w)+|w|^{\frac{-\beta}{1-\beta}}(\varepsilon|k|)^{\frac{\beta}{1-\beta}} \varepsilon^{2} \varphi(\varepsilon) p\right)^{2}+(w \cdot e)^{2}} \\
& \quad \times \frac{\widetilde{\nu}^{\varepsilon}(w) \widetilde{F}_{0}^{\varepsilon}(w)}{|w|^{\frac{N+\alpha-\beta}{1-\beta}}}(\varepsilon|k|)^{\frac{N+\alpha-\beta}{1-\beta}} \ell\left(\frac{|w|^{\frac{1}{1-\beta}}}{(\varepsilon|k|)^{\frac{1}{1-\beta}}}\right)(\varepsilon|k|)^{-\frac{N}{1-\beta}}|w|^{-\frac{N-\beta}{1-\beta}} d w,
\end{aligned}
$$

where

$$
\widetilde{\nu}^{\varepsilon}(w)=\widetilde{\nu}\left(\frac{w}{|w|^{\frac{-\beta}{1-\beta}}(\varepsilon|k|)^{\frac{1}{1-\beta}}}\right)
$$

and

$$
\widetilde{F}_{0}^{\varepsilon}(w)=\widetilde{F}_{0}\left(\frac{w}{|w|^{\frac{-\beta}{1-\beta}}(\varepsilon|k|)^{\frac{1}{1-\beta}}}\right)
$$

(we dropped the dependence in $k$ in $\widetilde{\nu}^{\varepsilon}$ and $\widetilde{F}_{0}^{\varepsilon}$ to keep notation simpler).

The fact that $\gamma=2$ now yields

$$
\begin{aligned}
& d_{2}^{\varepsilon}(p, k)= \\
& =\frac{(1-\beta)^{-1}}{\varphi(\varepsilon) \ln \left(\varepsilon^{-1}\right)}|k|^{2} \int_{|w| \geq M^{1-\beta} \varepsilon|k|} \frac{(w \cdot e)^{2}}{\left(\widetilde{\nu}^{\varepsilon}(w)+|w|^{\frac{-\beta}{1-\beta}}|k|^{\frac{\beta}{1-\beta}} \varphi(\varepsilon) \varepsilon^{\frac{\alpha}{1-\beta}} p\right)^{2}+(w \cdot e)^{2}} \\
& \quad \times \frac{\widetilde{\nu}^{\varepsilon}(w) \widetilde{F}_{0}^{\varepsilon}(w)}{|w|^{N+2}} \ell\left(\frac{|w|^{\frac{1}{1-\beta}}}{(\varepsilon|k|)^{\frac{1}{1-\beta}}}\right) d w .
\end{aligned}
$$

Lemma 5.1 below implies

$$
d_{2}^{\varepsilon}(p, k) \underset{\varepsilon \rightarrow 0}{\longrightarrow} \kappa|k|^{2}
$$

which concludes the proof of Theorem 3.4.

Lemma 5.1. We have:

$$
\begin{aligned}
& \frac{(1-\beta)^{-1}}{\varphi(\varepsilon) \ln \left(\varepsilon^{-1}\right)} \int_{|w| \geq M^{1-\beta} \varepsilon|k|} \frac{(w \cdot e)^{2}}{\left(\widetilde{\nu}^{\varepsilon}(w)+|w|^{\frac{-\beta}{1-\beta}}|k|^{\frac{\beta}{1-\beta}} \varphi(\varepsilon) \varepsilon^{\frac{\alpha}{1-\beta}} p\right)^{2}+(w \cdot e)^{2}} \\
& \quad \times \frac{\widetilde{\nu}^{\varepsilon}(w) \widetilde{F}_{0}^{\varepsilon}(w)}{|w|^{N+2}} \ell\left(\frac{|w|^{\frac{1}{1-\beta}}}{(\varepsilon|k|)^{\frac{1}{1-\beta}}}\right) d w \rightarrow \kappa
\end{aligned}
$$

as $\varepsilon \rightarrow 0$. 
Proof of Lemma 5.1: First, we show that

$$
\kappa:=\frac{1}{(1-\beta)} \lim _{\lambda \rightarrow 0} \frac{1}{\ln \left(\lambda^{-1}\right)} \int_{|w| \geq \lambda} \frac{w_{1}^{2}}{\nu_{0}^{2}+w_{1}^{2}} \frac{\kappa_{0} \nu_{0}}{|w|^{N+2}} d w
$$

is well defined: We denote

$$
\psi(\lambda)=\int_{|w| \geq \lambda} \frac{w_{1}^{2}}{\nu_{0}^{2}+w_{1}^{2}} \frac{\kappa_{0} \nu_{0}}{|w|^{N+2}} d w
$$

and then we have

$$
\begin{aligned}
\psi^{\prime}(\lambda) & =-\int_{|w|=\lambda} \frac{w_{1}^{2}}{\nu_{0}^{2}+w_{1}^{2}} \frac{\kappa_{0} \nu_{0}}{|w|^{N+2}} d \sigma(w) \\
& =-\frac{\kappa_{0} \nu_{0}}{\lambda^{N+2}} \int_{|w|=\lambda} \frac{w_{1}^{2}}{\nu_{0}^{2}+w_{1}^{2}} d \sigma(w) \\
& =-\frac{\kappa_{0} \nu_{0}}{\lambda} \int_{|v|=1} \frac{v_{1}^{2}}{\nu_{0}^{2}+\lambda^{2} v_{1}^{2}} d \sigma(v) .
\end{aligned}
$$

We deduce from L'Hopital's rule that

$\kappa=\frac{1}{(1-\beta)} \lim _{\lambda \rightarrow 0} \frac{\psi(\lambda)}{\ln \left(\lambda^{-1}\right)}=\frac{1}{(1-\beta)} \lim _{\lambda \rightarrow 0} \frac{\psi^{\prime}(\lambda)}{\ln ^{\prime}\left(\lambda^{-1}\right)}=\frac{\kappa_{0}}{(1-\beta) \nu_{0}} \int_{|v|=1} v_{1}^{2} d \sigma(v)$.

Then, we note that from the assumptions (B1-B2) and the definition of a slowly varying function, for any $w$ and $k$, we have

$$
\begin{aligned}
& \widetilde{\nu}^{\varepsilon}(w)=\nu_{0}+o(1) \quad \text { as } \varepsilon \rightarrow 0, \\
& \widetilde{F}_{0}^{\varepsilon}(w)=\kappa_{0}+o(1) \quad \text { as } \varepsilon \rightarrow 0,
\end{aligned}
$$

and

$$
\frac{1}{\varphi(\varepsilon)} \ell\left(\frac{|w|^{\frac{1}{1-\beta}}}{(\varepsilon|k|)^{\frac{1}{1-\beta}}}\right)=\frac{1}{\ell\left(\varepsilon^{-\frac{1}{1-\beta}}\right)} \ell\left(\frac{|w|^{\frac{1}{1-\beta}}}{(\varepsilon|k|)^{\frac{1}{1-\beta}}}\right)=1+o(1) \quad \text { as } \varepsilon \rightarrow 0 .
$$

Thus, the integrand in (48) divided by $\varphi(\varepsilon)$ converges pointwise to

$$
\frac{(w \cdot e)^{2}}{\nu_{0}^{2}+(w \cdot e)^{2}} \frac{\nu_{0} \kappa_{0}}{|w|^{N+2}} \quad \text { as } \varepsilon \rightarrow 0,
$$

and it is bounded by

$$
C \frac{(w \cdot e)^{2}}{\nu_{1}^{2}+(w \cdot e)^{2}} \frac{1}{|w|^{N+2}}
$$


Then, easy but tedious computations yield, for bounded $p$ and $k$ and for $\varepsilon$ going to 0 :

$$
\begin{aligned}
& \mid \int_{|w| \geq M^{1-\beta} \varepsilon|k|} \frac{(w \cdot e)^{2}}{\left(\widetilde{\nu}^{\varepsilon}(w)+|w|^{\frac{-\beta}{1-\beta}}|k|^{\frac{\beta}{1-\beta}} \varphi(\varepsilon) \varepsilon^{\frac{\alpha}{1-\beta}} p\right)^{2}+(w \cdot e)^{2}} \\
& \quad \times \frac{\widetilde{\nu}^{\varepsilon}(w) \widetilde{F}_{0}^{\varepsilon}(w)}{|w|^{N+2}} \ell\left(\frac{|w|^{\frac{1}{1-\beta}}}{(\varepsilon|k|)^{\frac{1}{1-\beta}}}\right) d w-\int_{|w| \geq M^{1-\beta} \varepsilon|k|} \frac{w_{1}^{2}}{\nu_{0}^{2}+w_{1}^{2}} \frac{\kappa_{0} \nu_{0}}{|w|^{N+2}} d w \mid \\
& \leq o(1) \int_{|w| \geq M^{1-\beta} \varepsilon|k|} \frac{w_{1}^{2}}{\nu_{0}^{2}+w_{1}^{2}} \frac{\kappa_{0} \nu_{0}}{|w|^{N+2}} d w \\
& \quad C \varphi(\varepsilon) \varepsilon^{\frac{\alpha}{1-\beta}} \int_{|w| \geq M^{1-\beta} \varepsilon|k|} \frac{w_{1}^{2}}{\nu_{0}^{2}+w_{1}^{2}} \frac{\kappa_{0} \nu_{0}}{|w|^{N+2+\beta /(1-\beta)}} d w \\
& \quad \leq o(1) \ln \left(\varepsilon^{-1}\right)+C \varphi(\varepsilon) \varepsilon^{2} \\
& \leq o(1) \ln \left(\varepsilon^{-1}\right) .
\end{aligned}
$$

Thus we have

$$
\begin{aligned}
& \lim _{\varepsilon \rightarrow 0} \frac{1}{\ln \left(\varepsilon^{-1}\right)} \int_{|w| \geq M^{1-\beta} \varepsilon|k|} \frac{(w \cdot e)^{2}}{\left(\widetilde{\nu}^{\varepsilon}(w)+|w|^{\frac{-\beta}{1-\beta}}|k|^{\frac{\beta}{1-\beta}} \varphi(\varepsilon) \varepsilon^{\frac{\alpha}{1-\beta}} p\right)^{2}+(w \cdot e)^{2}} \\
& \quad \times \frac{\widetilde{\nu}^{\varepsilon}(w) \widetilde{F}_{0}^{\varepsilon}(w)}{|w|^{N+2}} \ell\left(\frac{|w|^{\frac{1}{1-\beta}}}{(\varepsilon|k|)^{\frac{1}{1-\beta}}}\right) d w \\
& \quad=\lim _{\varepsilon \rightarrow 0} \frac{1}{\ln \left(\varepsilon^{-1}\right)} \int_{|w| \geq M^{1-\beta} \varepsilon|k|} \frac{w_{1}^{2}}{\nu_{0}^{2}+w_{1}^{2}} \frac{\kappa_{0} \nu_{0}}{|w|^{N+2}} d w .
\end{aligned}
$$

Finally, using the fact that for all $k \neq 0$,

$$
\lim _{\varepsilon \rightarrow 0} \frac{\ln \left(\left(M^{1-\beta} \varepsilon|k|\right)^{-1}\right)}{\ln \left(\varepsilon^{-1}\right)}=1,
$$

we deduce (49), which completes the proof of Lemma 5.1,

\section{Appendix}

Lemma 6.1. Let us assume that $b=b\left(v, v^{\prime}\right)=\left|v-v^{\prime}\right|^{\beta}$ with $\beta \in \mathbb{R}$.

(i) For $\beta \in(-N, \alpha)$ there exists $C$ such that

$$
C^{-1}\langle v\rangle^{\beta} \leq \nu(v):=K(F)(v) \leq C\langle v\rangle^{\beta}, \quad \forall v \in \mathbb{R}^{N} .
$$


(ii) For $\beta \in(-\min \{\alpha ; N\}, \min \{\alpha ; N\})$ there exists $M$ such that

$$
\int_{\mathbb{R}^{N}} F^{\prime} \frac{\nu}{b} d v^{\prime} \leq M, \quad \forall v \in \mathbb{R}^{N}
$$

(iii) For $\beta \in(-\min \{\alpha ; N / 2\}, \alpha)$ there exists $M$ such that

$$
\int_{\mathbb{R}^{N}} \frac{F^{\prime}}{\nu^{\prime}} \frac{b^{2}}{\nu^{2}} d v^{\prime} \leq M \quad \forall v \in \mathbb{R}^{N} .
$$

Proof of Lemma 6.1. Point (i). The case $\beta \geq 0$ being very simple, we only deal with the case $\beta<0$ and large values of $|v|$. We split $\nu=\nu_{1}+\nu_{2}$ with

$\nu_{1}(v)=\int_{\left|v^{\prime}-v\right| \leq|v| / 2} F\left(v^{\prime}\right)\left|v-v^{\prime}\right|^{\beta} d v^{\prime}, \quad \nu_{2}(v)=\int_{\left|v^{\prime}-v\right| \geq|v| / 2} F\left(v^{\prime}\right)\left|v-v^{\prime}\right|^{\beta} d v^{\prime}$.

On the one hand

$$
\nu_{1}(v) \leq F(|v| / 2) \int_{\left|v^{\prime}-v\right| \leq|v| / 2}\left|v-v^{\prime}\right|^{\beta} d v^{\prime} \leq F_{0}|v|^{-\alpha-N}|v|^{N+\beta} \leq C|v|^{\beta} .
$$

On the other hand

$$
\nu_{2}(v) \leq(|v| / 2)^{\beta} \int_{\left|v^{\prime}-v\right| \geq|v| / 2} F^{\prime} d v^{\prime} \leq C|v|^{\beta} .
$$

Finally,

$\nu_{2}(v) \geq(3|v| / 2)^{\beta} \int_{|v| / 2 \leq\left|v^{\prime}-v\right| \leq 3|v| / 2} F^{\prime} d v^{\prime} \geq(3|v| / 2)^{\beta} \int_{|v| \leq 1} F^{\prime} d v^{\prime} \geq C^{-1}|v|^{\beta}$

for $|v| \geq 2$.

Points (ii) and (iii). From Lemma 6.1 we have on the one hand

$$
\int_{\mathbb{R}^{N}} \frac{F^{\prime}}{b} d v^{\prime}=\int_{\mathbb{R}^{N}} F^{\prime}\left|v-v^{\prime}\right|^{-\beta} \leq C\langle v\rangle^{-\beta} \leq M \nu^{-1} \quad \forall v \in \mathbb{R}^{N},
$$

and on the other hand

$$
\int_{\mathbb{R}^{N}} \frac{F^{\prime}}{\nu^{\prime}} b^{2} d v^{\prime} \leq \int_{\mathbb{R}^{N}}\left\langle v^{\prime}\right\rangle^{-N-\alpha-\beta}\left|v-v^{\prime}\right|^{2 \beta} \leq C\langle v\rangle^{2 \beta} \leq M \nu^{2}, \quad \forall v \in \mathbb{R}^{N} .
$$

Acknowledgements: We thank Jean Dolbeault and Stefano Olla for fruitful discussions during the preparation of this work. 


\section{References}

[1] (MR0743736) C. Bardos, R. Santos and R. Sentis, Diffusion approximation and computation of the critical size, Trans. A. M. S., 284 (1984), 617-649.

[2] (MR0533346) A. Bensoussan, J. L. Lions and G. Papanicolaou, Boundary layers and homogenization of transport processes, Publ. Res. Inst. Math. Sci. 15 (1979), $53-157$.

[3] (MR1015093) N.H. Bingham, C.M. Goldie and J.L. Teugels, Regular variation, Encyclopedia of Mathematics and its Applications 27. Cambridge University Press, Cambridge, 1989.

[4] (MR1749231) A.V. Bobylev, J.A. Carrillo and I.M. Gamba, On some properties of kinetic and hydrodynamic equations for inelastic interactions, J. Statist. Phys. 98 (2000), 743-773.

[5] (MR2264617) A.V. Bobylev and I.M. Gamba, Boltzmann equations for mixtures of Maxwell gases: exact solutions and power like tails, J. Stat. Phys. 124 (2006), $497-516$.

[6] (MR1174046) C. Börgers, C. Greengard and E. Thomann, The Diffusion Limit of Free Molecular Flow in Thin Plane Channels, SIAM J. Appl. Math. 52 (1992), 1057-1075.

[7] (MR1803225) P. Degond, T. Goudon and F. Poupaud, Diffusion limit for non homogeneous and non-micro-reversibles processes, Indiana Univ. Math. J. 49 (2000), 1175-1198.

[8] (MR1649918) C. Dogbe, Diffusion Anormale pour le Gaz de Knudsen. C. R. Acad. Sci. Paris Sér. I Math. 326 (1998), 1025-1030.

[9] (MR1788479) C. Dogbe, Anomalous diffusion limit induced on a kinetic equation, J. Statist. Phys. 100 (2000), 603-632.

[10] D. Duering and G. Toscani, Anomalous diffusion limit indeuced on a kinetic equation, Physica A 384 (2007), 493-506.

[11] (MR1942001) M.H. Ernst, and R. Brito, Scaling solutions of inelastic Boltzmann equations with over-populated high energy tails, J. Statist. Phys. 109 (2002), 407432.

[12] (MR1632712) F. Golse, Anomalous Diffusion Limit for the Knudsen Gas, Asymptotic Anal. 17, (1998), 1-12.

[13] H. Jara, T. Komorowski and S. Olla, A limit theorem for additive functionals of a Markov chain, Preprint arXiv:0809.0177.

[14] (MR0339741) E.W. Larsen and J.B. Keller, Asymptotic solution of neutron transport problems for small mean free paths, J. Math. Phys. 15 (1974), 75-81.

[15] A. Mellet and B. Texier, work in preparation.

[16] D.A. Mendis and M. Rosenberg, Cosmic dusty plasma, Annu. Rev. Astron. Astrophys. 32 (1994), 419-63.

[17] M.E.J. Newman, Power laws, Pareto distributions and Zipf's law, Contemp. Phys. 46 (2005), 323-351.

[18] D. Summers and R.M. Thorne, The modified plasma dispersion function, Phys. Fluids 83 (1991), 1835-1847. 
[19] (MR2264625) C. Villani, Mathematics of granular materials, J. Stat. Phys. 124 (2006), 781-822.

[20] E. Wigner, Nuclear reactor theory, AMS (1961).

[21] I. Wright, The social architecture of capitalism, Physica A 346 (2005), 589-620. 